University of Redlands

\title{
U.S. Air Force Thunderbirds \\ Routine Mapping Application
}

A Major Individual Project submitted in partial satisfaction of the requirements

for the degree of Master of Science in Geographic Information Systems

by

Alex R. Strobl

Ruijin Ma, Ph.D., Committee Chair

Mark Kumler, Ph.D.

May 2020 
U.S. Air Force Thunderbirds

Routine Mapping Application

Copyright (C) 2020

by

Alex R. Strobl 
The report of Alex R. Strobl is approved.

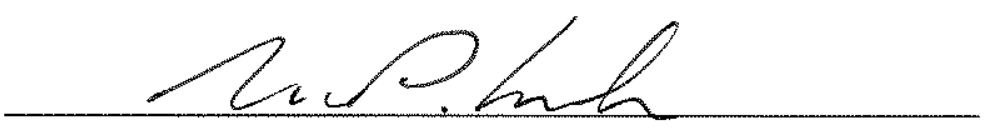

Mark Kumler, Ph.D.

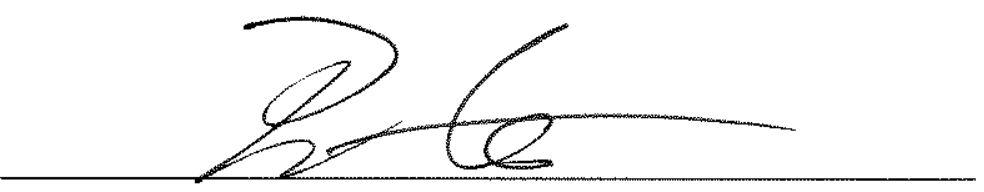

Ruijin Ma, Ph.D., Committee Chair

May 2020 



\section{Acknowledgements}

First and foremost, I would like to express my gratitude and love for my parents, siblings, and the rest of my family for their support through this exciting and difficult experience in my life. Thank you to my parents Robert and Kerri for encouraging me to pursue my goals and dreams. To my sister Brittany and my brother Andrew, thank you for always being there for me and helping me get through the stress of this program. To the rest of my family, your unconditional love and words of encouragement mean the world to me. I am truly grateful that I have such an amazing family.

I would also like to thank my advisor, Dr. Ruijin Ma, who spent this year pushing me and guiding me towards a better thesis. Your expertise and knowledge really helped me meticulously think through every aspect of this project. Thank you to Dr. Mark Kumler for your interest in my project and for taking the time to be my second reader.

This project would not be possible without client, Mr. Fon Allen Duke and the Mojave Desert Ecosystem Program (MDEP). Thank you for bringing such a unique and awesome project to the table. A special thanks to Mr. Jason Boggs from MDEP, who set up the server for my project and spent a lot of time helping me make sure all of the different aspects of the application come together. I would not have finished without your help. A big thanks to Shilpi for helping me understand my least favorite language, PHP.

Finally, I would like to thank everyone from MS GIS cohorts 25.5, 26, 27, and 27.5. We all worked very hard this past year and survived this rollercoaster together. I have made some great friends from these cohorts and I am proud of what we accomplished as a group. A special shout out to Rene, Matt, Pat, Neel, Colin, David, and Bryce for all of the game nights, FIFA, golf, and general shenanigans that helped me survive this year. 



\section{Abstract \\ U.S. Air Force Thunderbirds Routine Mapping Application \\ by}

Alex R. Strobl

The U.S. Air Force Thunderbirds is a military demonstration group that performs aerial acrobatic stunts at air shows around the world. The group of six planes and over 120 support staff meticulously plan every air show to get a general layout of the surroundings and look for potential hazards. In the past, paper maps were used to aid the group in planning their routine, but they now seek an easier way to map and display their data. With the use of open source software, a web application was created that gives the planning staff and pilots the ability to dynamically create, edit, and view airshow routine maps on their mobile devices. The resulting web application allows for a more precise mapping process, easier editing and access, and hosts the data in a 3 dimensional environment for safety analysis. 



\section{Table of Contents}

Chapter 1 - Introduction ......................................................................................... 1

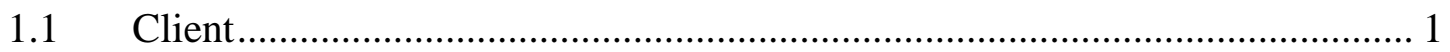

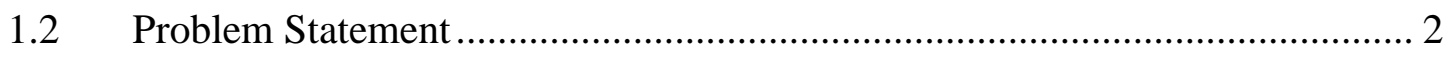

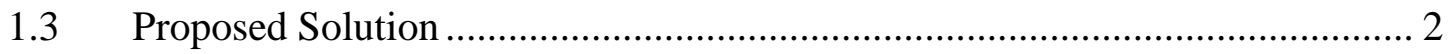

1.3.1 Goals and Objectives ........................................................................... 2

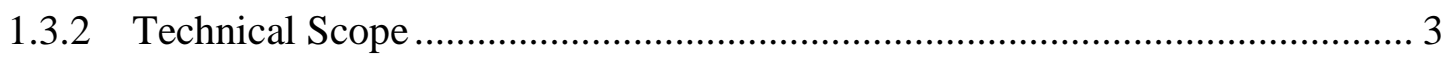

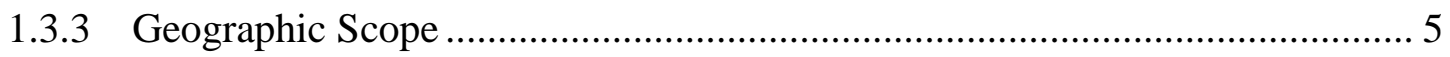

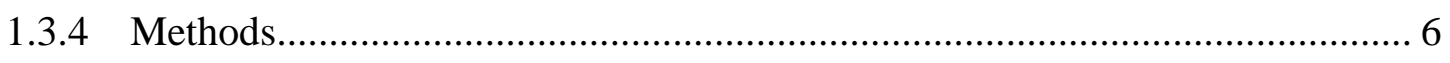

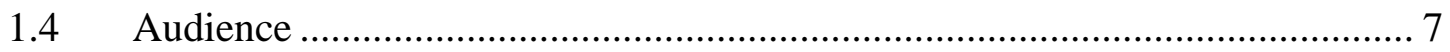

1.5 Overview of the Rest of this Report ..................................................... 8

Chapter 2 - Background and Literature Review ............................................................... 9

2.1 United States Air Force Thunderbirds ……………................................... 9

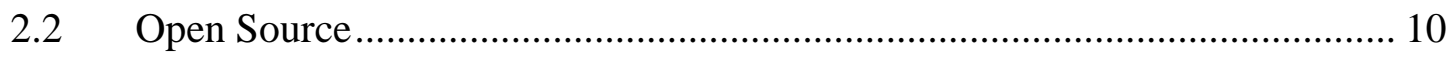

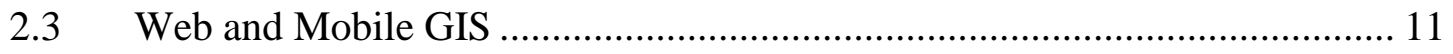

2.4 Planning and Modeling with GIS ............................................................. 13

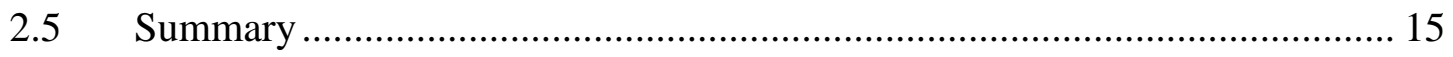

Chapter 3 - Systems Analysis and Design......................................................................... 17

3.1 Problem Statement .................................................................................. 17

3.2 Requirements Analysis ............................................................................. 17

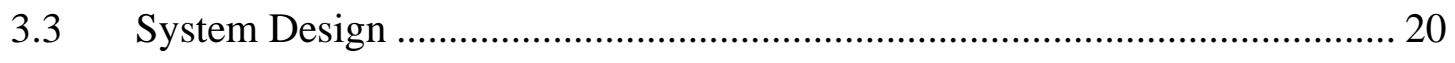

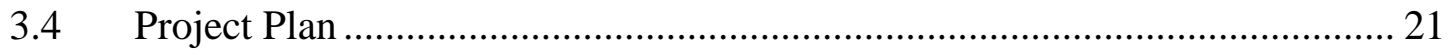

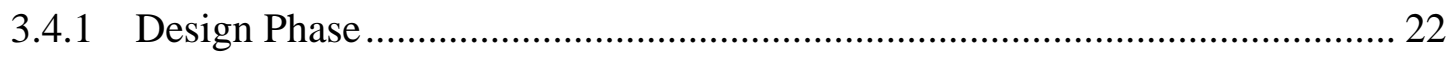


3.4.2 Development Phase ..................................................................................... 23

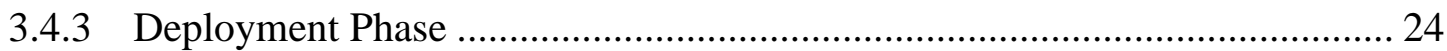

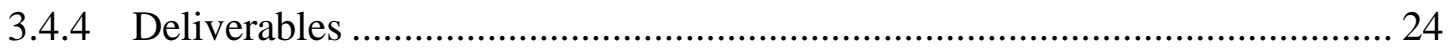

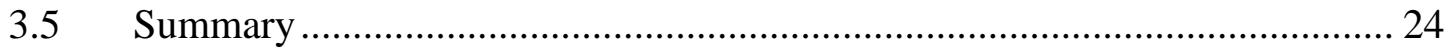

Chapter 4 - Database Design..................................................................................................... 27

4.1 Conceptual Data Model ..................................................................... 27

4.2 Logical Data Model .......................................................................... 28

4.3 Data Sources, Scrubbing, and Migration …………………………............... 30

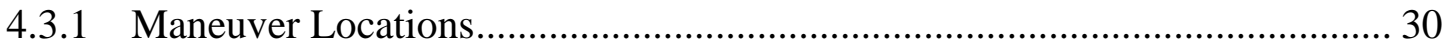

4.3.2 Building Obstruction Data .......................................................................... 31

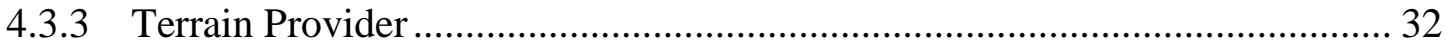

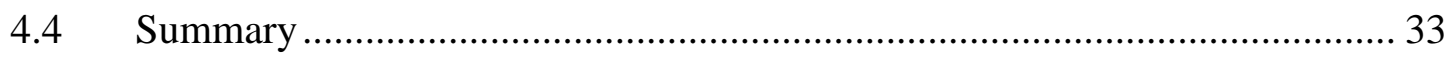

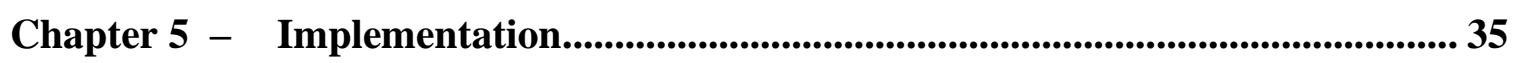

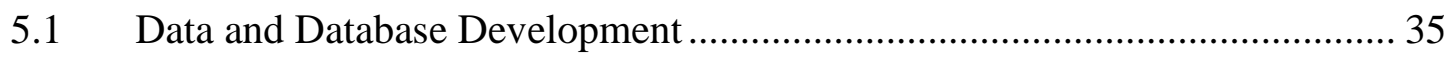

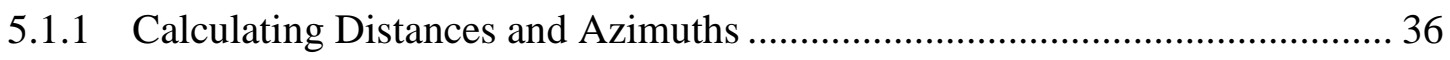

5.1.2 Creating a New Database ............................................................................ 37

5.1.3 Creating Spatially Enabled Data Tables …………………………….......... 38

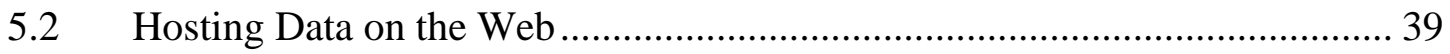

5.2.1 Setting up the Workspace ........................................................................... 40

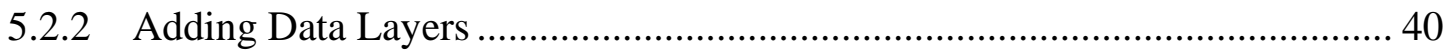

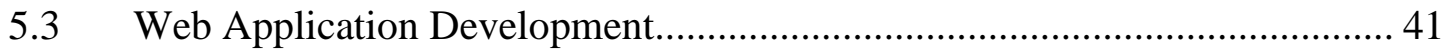

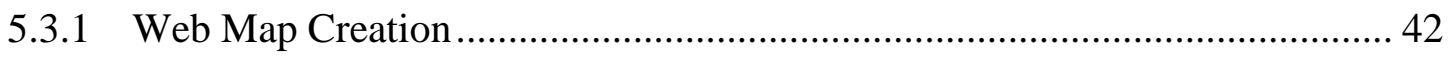

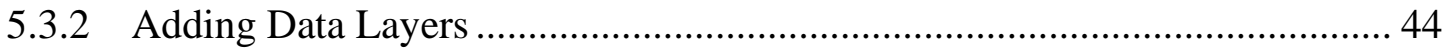




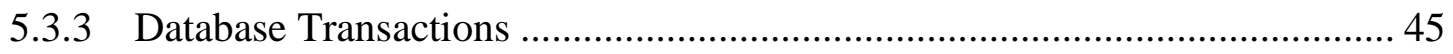

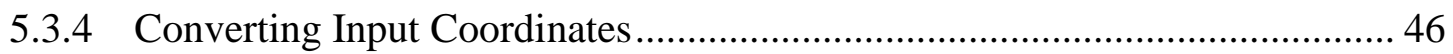

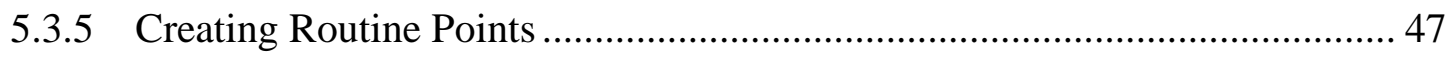

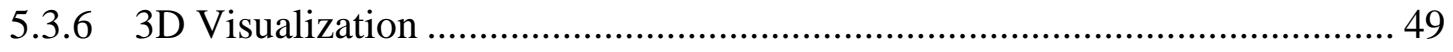

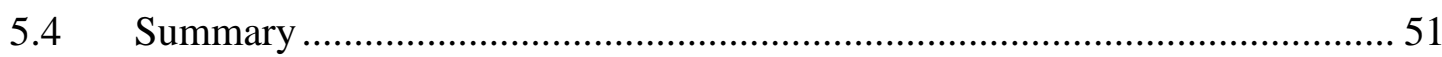

Chapter 6 - Results and Analysis........................................................................................ 53

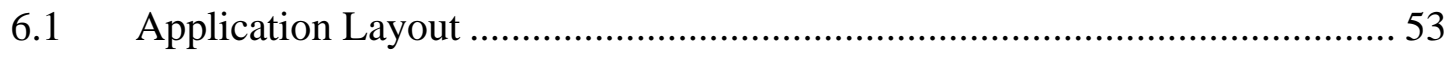

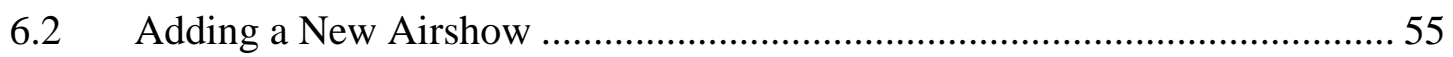

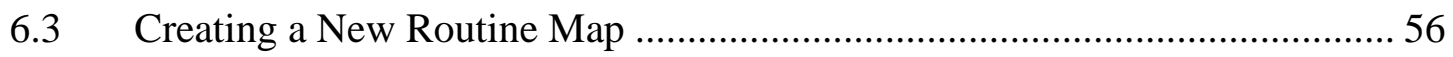

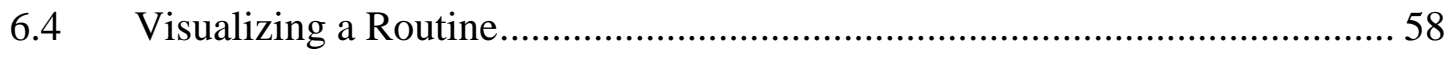

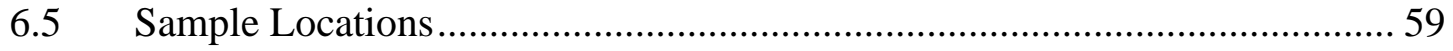

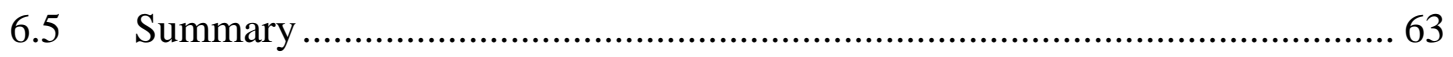

Chapter 7 - Conclusions and Future Work ...........................................................65

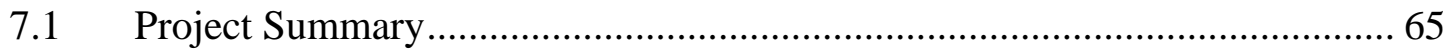

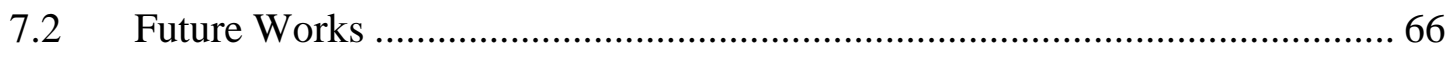

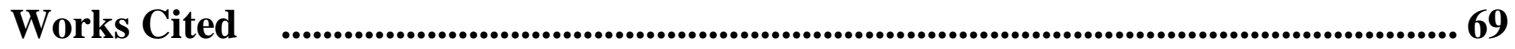

Appendix A. Sample Thunderbirds Routine Maps ............................................. 71

Appendix B. Sample Thunderbirds Routine Maps .................................................... 72

Appendix C. Distance and Angles Python Script.............................................. 73

Appendix D. OpenLayers 3 JavaScript Functions ..................................................... 74

Appendix E. Cesium JavaScript Functions............................................................ 84

Appendix F. User Guide....................................................................................................... 86 



\section{Table of Figures}

Figure 1-1: Sample Airshow Map at Nellis Air Force Base ..................................6

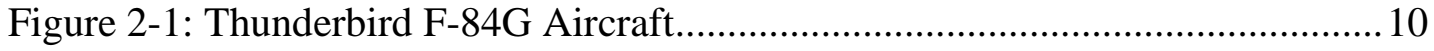

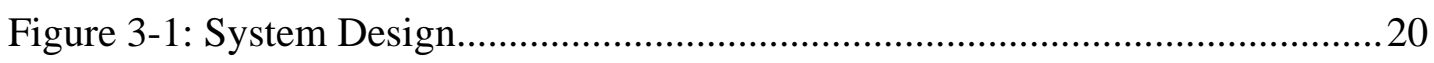

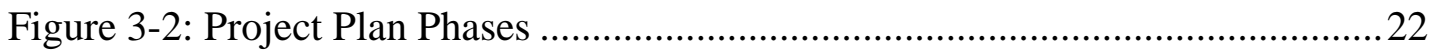

Figure 4-1: Conceptual Data Model ............................................................. 28

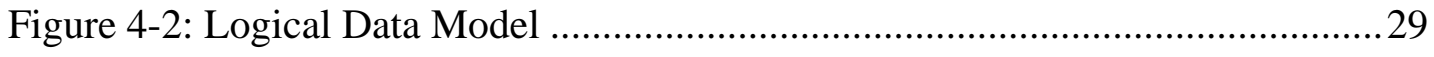

Figure 4-3: Terrain Height Sample Spacing ................................................... 32

Figure 5-1: Azimuth and Great Circle Distance Representations............................37

Figure 5-2: Database Layout in pgAdmin.. ...................................................... 38

Figure 5-3: Sample Code for Inserting Data into a Table in PostgreSQL .................39

Figure 5-4: GeoServer Setup for the Application ............................................. 40

Figure 5-5: Thunderbirds Routine Mapping Application Workflow Model ............. 42

Figure 5-6: Basic Web Map with Bing Maps Satellite Imagery............................. 44

Figure 5-7: Symbology and Style for Data Layers .......................................... 45

Figure 5-8: Sample Code for Creating a Transaction Function ...............................46

Figure 5-9: Maneuver Point Creation Process Model.............................................. 47

Figure 5-10: Model for Creating 3D Buildings in Airshow Extent .........................50

Figure 6-1: Thunderbirds Routine Mapping Application Homepage ........................54

Figure 6-2: Input Forms for Creating New Airshow and Bomb Burst Locations .....55

Figure 6-3: Airshow Selection Menu and Create/Clear Routine Buttons..................56

Figure 6-4: Nellis AFB Routine Map. ...............................................................57

Figure 6-5: Application Layers Menu.............................................................58 
Figure 6-6: Airshow Routine Displayed in 3D Environment................................ 59

Figure 6-7: Comparison of Application and Sample at Nellis AFB ........................ 60

Figure 6-8: Comparison of Application and Sample at Creech AFB ...................... 61

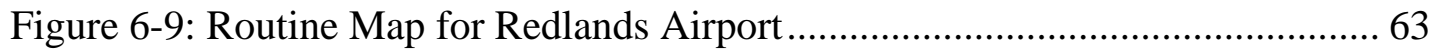




\section{List of Tables and Equations}

Table 3-1 Thunderbirds Aplication Requirements. ................................................. 19

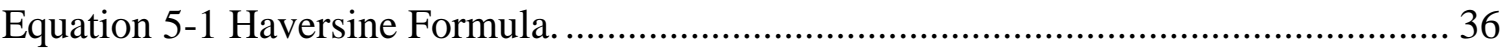

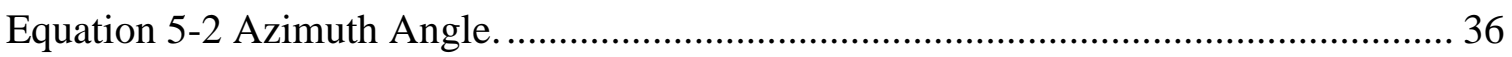

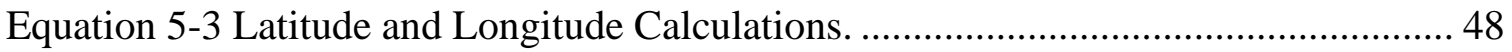





\section{List of Acronyms and Definitions}

\begin{tabular}{|c|c|}
\hline AFB & Air Force Base \\
\hline AGI & Analytical Graphics, Inc. \\
\hline API & Application Program Interface \\
\hline Bomb Burst & Flight Maneuver in Thunderbirds Routine \\
\hline CAC & Common Access Card \\
\hline CSS & Cascading Style Sheet \\
\hline DEM & Digital Elevation Model \\
\hline DOF & Digital Obstruction File \\
\hline ESRI & Environmental Systems Research Institute \\
\hline FAA & Federal Aviation Administration \\
\hline GIS & Geospatial Information System \\
\hline HTML & HyperText Markup Language \\
\hline ICAO & International Civil Aviation Organization \\
\hline KML & Keyhole Markup Language \\
\hline MDEP & Mojave Desert Ecosystem Program \\
\hline NAVD 88 & North American Vertical Datum of 1988 \\
\hline NGA & National Geospatial-Intelligence Agency \\
\hline PHP & Hypertext Preprocessor \\
\hline Primitive & Basic Geometry Object \\
\hline SOAP & Simple Object Access Protocol \\
\hline SQL & Standard Query Language \\
\hline TIN & Triangulated Irregular Network \\
\hline
\end{tabular}




$\begin{array}{ll}\text { WCS } & \text { Web Coverage Service } \\ \text { WFS } & \text { Web Feature Service } \\ \text { WFS-T } & \text { Web Feature Service - Transaction } \\ \text { WGS84 } & \text { World Geodetic System of } 1984 \\ \text { WMS } & \text { Web Mapping Service } \\ \text { WPS } & \text { Web Processing Service }\end{array}$




\section{Chapter 1 - Introduction}

The United States Air Force Thunderbirds is a special squadron of F-16 fighter jets that conducts aerial acrobatics for airshows and other demonstration purposes. The demonstration unit was established in 1953 with only four jets, but now features six jets, six support officers, and over 120 enlisted support personnel (United States Air Force, 2015). A lot of work goes into the preparations, planning, and routine for each airshow. While the specific routine of acrobatics is the same for every show, the pilots and support officers must take geographic data, such as terrain elevation and proximity to mountains, and city infrastructure into account. The Thunderbirds team traditionally completed all of this planning using paper maps, but after recently switching to Google Earth, they realized the limitations of this method and the benefits of switching to a digital format.

Converting from the paper method to a digital map creation process would give the Thunderbirds an easier way of presenting, storing, and accessing their airshow maps. The Thunderbirds expressed a desire to access and create routine maps while at the airshow on their mobile devices. To meet this goal, and in an effort to reduce map production time, this project aimed to create a mobile web application that will allow the Thunderbirds to create new routine maps, access and edit previously created maps, and identify some potential aerial hazards.

\subsection{Client}

While this project was created for use by the Thunderbirds, the client for this project was Mojave Desert Ecosystem Program (MDEP) and the point of contact was Mr. Fon Allen Duke, Project Manager. The MDEP is a government agency responsible for compiling 
and maintaining spatial information for the Mojave Desert area and providing spatially related services and solutions to other government agencies in the region (Mojave Desert Ecosystem Program, 2015). Mr. Duke was contacted by the U.S. Air Force Thunderbirds, who was looking for a GIS solution to help the pilots plan their flight routines at various locations. In addition to supplying technical assistance on open source platforms and server administration, MDEP also obtained and provided spatial data needed about the flight routine for the project and are initially hosting all solutions for the Thunderbirds on its web servers. MDEP will work with the Thunderbirds to transfer the solutions to its own servers.

\subsection{Problem Statement}

The paper map system that the pilots had been using for planning their routines at various locations had become too cumbersome. In addition to time constraints, the pilots faced problems with visualizing the entire routine on one paper map and were unable to quickly edit the map if a potential hazard was found or a point location needed to be changed.

\subsection{Proposed Solution}

The proposed solution to the problem set forth by the U.S. Air Force Thunderbirds and MDEP was to create a web application to allow the pilots and planning staff to create, edit, and revisit airshow routines at different venues around the world from their mobile devices.

\subsubsection{Goals and Objectives}

The main goal of this project was to deliver a mobile web application that allows the U.S. Air Force Thunderbird pilots and planning staff to easily create, edit, and access their 
airshow routine maps while also allowing for analysis of the surrounding area for potential threats to the aircraft and pilots. This web application had to work on mobile devices such as the iPad or Windows Notepad. In order to meet this goal, the following objectives were established:

- Develop a geospatially enabled database for storing all geographic information about airshow locations and routine points

- Script a JavaScript tool that calculates routine point locations based on the user input for the show center location and the location of the acrobatic "Bomb Burst" stunt

- Develop a web application that can be accessed through mobile devices, accepts user inputs, allows for routine map editing, and displays geographic information for routine points, true headings and buildings that potentially pose a threat to pilot safety

- Integrate all geographic information into a three dimensional (3D) environment for routine safety analysis

\subsubsection{Technical Scope}

This project was completed using the OpenGeo Suite by Boundless. The components of the OpenGeo Suite used include PostGIS/pgAdmin III for the creation of geospatially enabled Structured Query Language (SQL) databases, GeoServer for hosting and publishing the data and services, and OpenLayers 3 JavaScript Application Program Interface (API) for the protocol used to generate the mobile web application. The OpenLayers 3 Cesium extension API was also used to implement the 3D environment 
provided in the application. The OpenGeo Suite was used at the request of the MDEP as they explore the functionality offered by open source software.

The sole deliverable for this project was a mobile web application that allows the Thunderbirds pilots and support staff to create, access, edit, and analyze flight routine maps for their airshows anywhere in the United States. Any device that has access to the internet or cellular data and a working web browser can connect to the application. This application relies on the databases created in PostGIS, the services hosted with GeoServer, and user input information for new airshow locations. In accordance with U.S. Air Force practices, all coordinates must be given and displayed in degrees and decimal minutes. After the user enters the coordinates for the show center location and the acrobatic "Bomb Burst" location, the application automatically calculates and displays the location of the rest of the flight routine based on predetermined routine specifications provided by the Thunderbirds. True headings are also calculated and displayed in the web application. After a map is created, the application has options for saving the map as a JPEG image file.

In addition to displaying routine point locations and magnetic headings, the application also displays location information for buildings that are identified as potential obstructions and has a digital terrain model so that the Thunderbirds can look for potential aerial threats. If there is an obstruction conflict, the application allows for editing of the routine map so that the routine will avoid these areas. Finally, the application can be integrated into a 3D environment so that the pilots can explore and analyze the routine in greater detail. 


\subsubsection{Geographic Scope}

While this application has the ability to create a routine map at any point in the world, the geographic scope of this project was the United States. This allowed for smaller datasets and faster application response time. For the purpose of this report, three sample locations were used for analyzing and testing the applications abilities.

- Nellis Air Force Base (AFB), the home location of the Thunderbirds, located near Las Vegas, Nevada. A sample airshow map of Nellis AFB was provided with the data from the Thunderbirds (see Figure 1-1 for sample and Appendix A for the full routine map)

- Creech AFB: Creech AFB is located in Indian Springs, Nevada. The Thunderbirds also provided a sample routine map for this location (See Appendix B)

- Redlands Airport: Redlands Airport is a small airfield located in Redlands, CA. This site was chosen for its proximity to nearby mountains and because the airfield hosts an annual airshow 


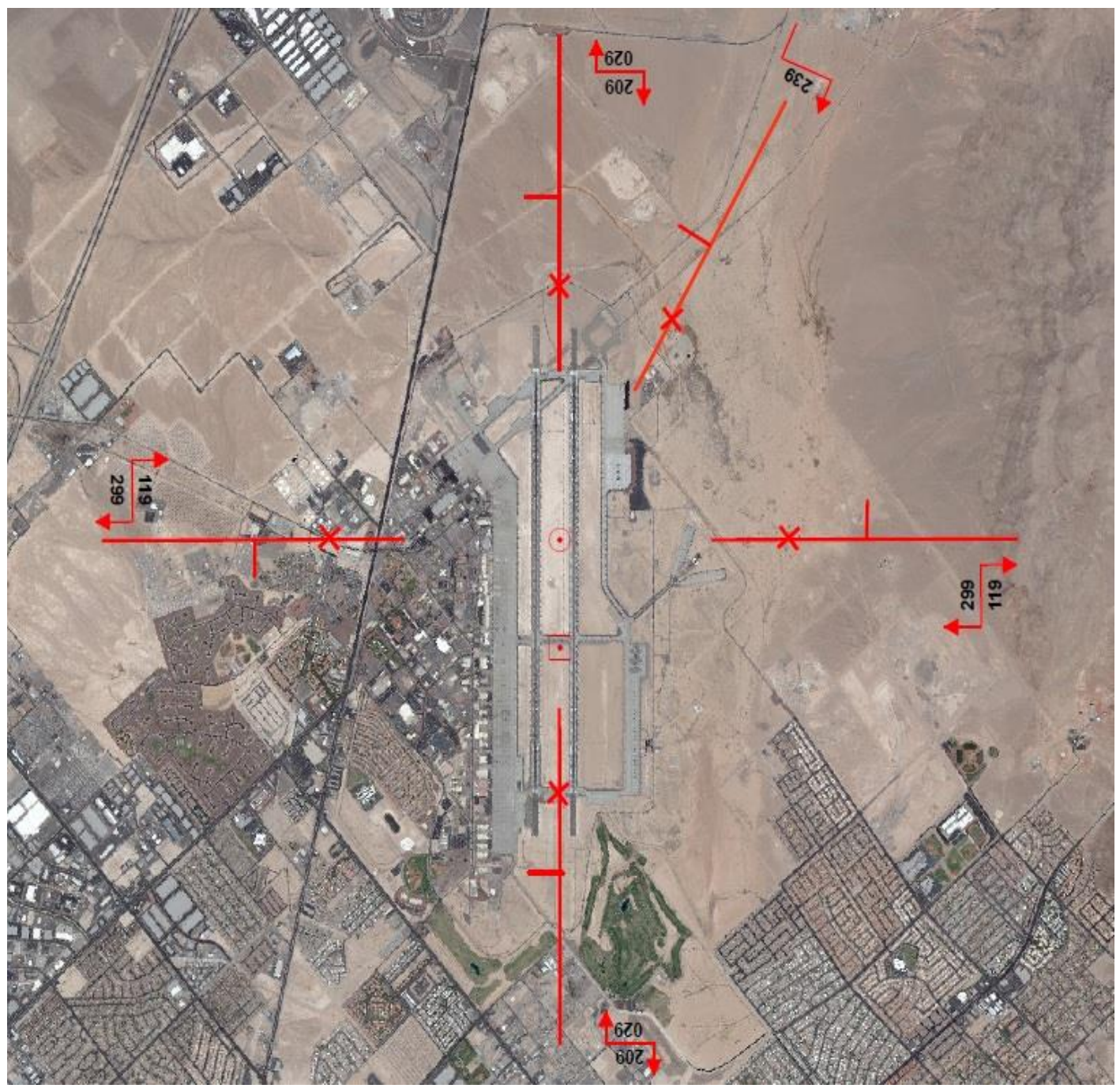

\section{Figure 1-1: Sample Airshow Map of Nellis Air Force Base}

\subsubsection{Methods}

In order to successfully integrate the data and provide a complete application, the following methodologies were used for the final product.

The mobile web application was developed with the use OpenGeo suite including GeoServer and PostGIS. PostGIS allowed for the creation of spatially enabled tables while GeoServer hosted the data to be used in the application. PostGIS was used for storing information used in and added by the user. Four datasets were used in the final database structure. The datasets for aerial obstructions were hosted on the server for 
Nevada and California, but were clipped to specific air show locations and tiled for easier, faster access and loading in the web application. The application was scripted with the use of JavaScript, HTML5, PHP, and CSS. OpenGeo's OpenLayers 3 API was used to provide the protocol needed to integrate geographic functions and services into the application. The OpenLayers 3 Cesium extension was used also to create and integrate a 3D environment into the application. This extension also came with an API that was used to incorporate the terrain model and extrude the buildings dataset.

In order to create the routine maneuver locations, a Python script and a JavaScript function were created. The first script used test data provided by the Thunderbirds to calculate the angle and distance of each maneuver location relative to the show center and "Bomb Burst" points. The results from this tool were used in the second script, which calculates maneuver locations based on a show center and its "Bomb Burst" location. In order to give users the ability to add and edit data that was hosted in GeoServer, the datasets were provided as a Web Feature Service (WFS), which allows the application to communicate with the server through a WFS - Transaction (WFS-T). This feature service functionality was accessed through a JavaScript function in the application script.

\subsection{Audience}

The users of this application will mostly be non-technical GIS users, and a separate instructional manual was written to explain the use cases and functionality in more detail. Since this report discusses the theory and application of GIS technology, it is mostly written to satisfy the interests of GIS users, both non-professional and professional. This report can also meet the learning interests of anyone who is looking for more information about the use of the OpenGeo suite. 


\subsection{Overview of the Rest of This Report}

The rest of this report explores the more in-depth theories and methodology used to complete the Thunderbirds Mobile Web Application. Chapter 2 delves into the background of the project through research on similar technological applications and geospatial theory. Chapter 3 focuses on the system design and project plan. Chapter 4 provides more detail on the conceptual and logical model designs and the data used for the project. Chapter 5 discusses the implementation process selected for this process. Chapter 6 examines the use cases of the application. Chapter 7 concludes the report and discusses potential future work to improve upon this application. 


\section{Chapter 2 - Background and Literature Review}

In order to devise a proper project solution that satisfies all requirements, a thorough background investigation needs to be completed to aid in the decision making process. GIS can be a powerful tool, but all important information regarding the situation needs to be taken into account. This chapter explores some of the concepts behind the Thunderbirds Routine Mapping Application, including a discussion of the Thunderbirds, aviation and GIS, and open source software.

\subsection{United States Air Force Thunderbirds}

The United States Air Force Thunderbirds is special squadron of F-16 fighter jets that conduct aerial acrobatics at airshows and other demonstrations. The unit was established in 1953 with only four jets, but now features six jets and pilots, six support officers, and over 120 enlisted support personnel (United States Air Force, 2015). When the team was founded, the F-84G jet (Figure 2-1) was selected as the official aircraft, since the straightwing configuration of the jet was well adapted for maneuvers and handling, but could not exceed the speed of sound (United States Air Force, 2015). The Thunderbirds have used seven different official aircraft throughout their history, and currently fly the F-16C.

While most of these changes were due to progressing technology, others were the result of unfortunate circumstances. Due to a gas crisis in 1973, the group switched to a noncombat aircraft, the T-38 Talon, to save on fuel. The Thunderbirds then changed from the T-38 Talon to the F-16 after multiple jets malfunctioned, causing all four planes to crash (Thunderbirds History, 2015). There have been several accidents in the squad's history, giving cause to why safety and planning are essential for each airshow. 


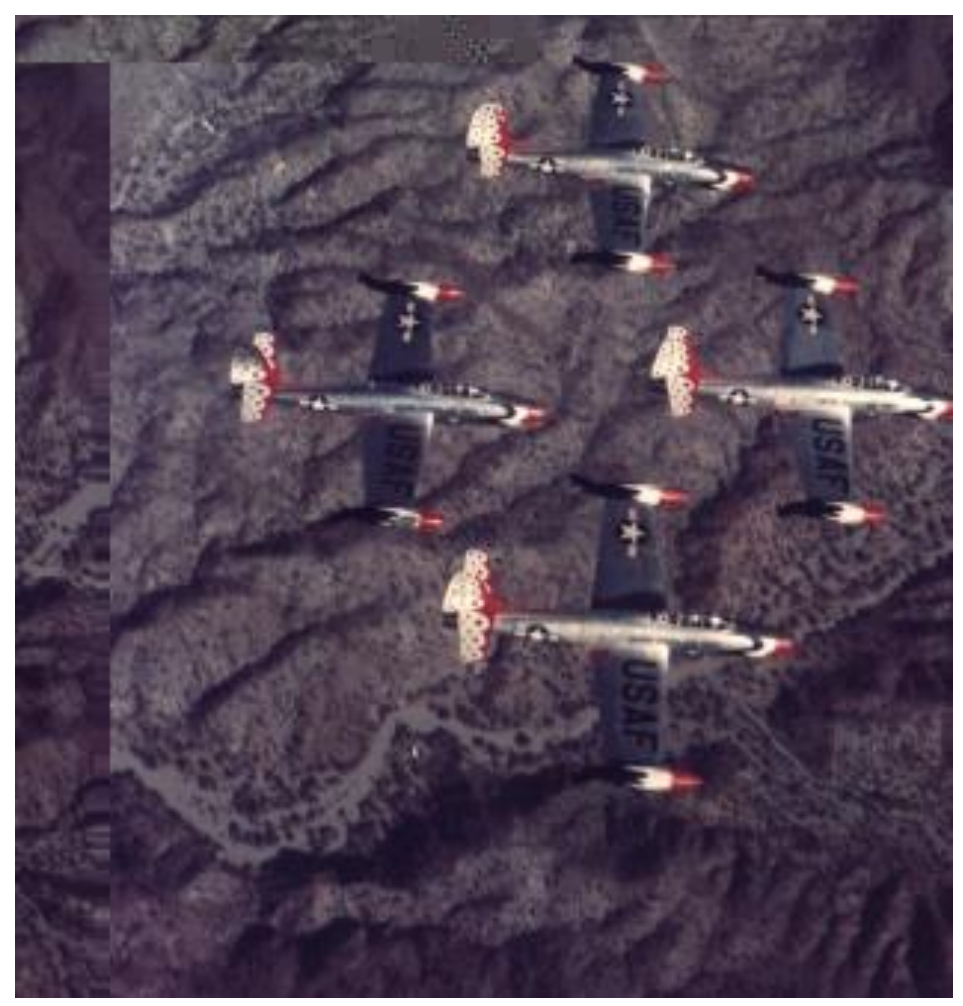

Figure 2-1: Thunderbird F-84G Aircraft

\subsection{Open Source}

Open source software, defined as "software where the source code is made available under a license that allows the modification, and re-distribution of the software at will," (OSGeo FAQ, 2015) plays an important role in the GIS professional world. The software is usually free for commercial use and allows for more customization, but at the expense of ease and time when compared to proprietary software. Many programs can be open source, including GIS, programming languages, and database management. Proprietary and open source software can usually be intermixed for specific functionality. In fact, the proprietary software company Environmental Systems Research Institute (ESRI) recommends a hybrid model of their software, encouraging the use of open source to 
“enhance users’ ESRI experience” (ArcNews Spring, 2011). The company often integrates open source components into its software packages.

OpenGeo Suite is an example of an open source software package. OpenGeo, provided by open source organization Boundless, is a collection of software used for the management of GIS data and creation of maps and geospatial applications. OpenGeo offers functionality and capabilities similar to the ESRI Suite, but is primarily web based and user-driven through scripting. This suite uses PostGIS and PostgreSQL to create and manage geospatial databases: GeoServer for hosting data online, GeoExplorer for composing maps, GeoWebCache for caching data and images, and OpenLayers and Boundless SDK for building custom web map applications. QGIS can also be used as a desktop application for analyzing geospatial data and creating maps (Education Center Boundless, 2015). Once the suite is installed on a user's server, the software can be integrated for data sharing and editing.

\subsection{Web and Mobile GIS}

Since the invention of the internet in 1969 , the world has embraced the ability to access information simply by searching in a web browser. It only makes sense that the same would hold true when GIS was introduced to the web. Web GIS, defined as any GIS process that uses the internet to communicate between components, has become extremely popular for its ability to reach a worldwide range of users and provides better cross-platform functionality because a simple web browser is all that is required (Fu \& Sun, 2011). This has become more apparent with advancements in mobile technologies. With the addition of positioning systems, wireless internet, and network capabilities, 
users can now access the internet and its applications through their phones and other mobile devices.

Advancements in mobile computing have given many companies and GIS users the ability to access, edit, and collect data in the field. This has allowed users to spend more time in the field as opposed to in an office, digitizing data collected on paper maps. Even with these technological advancements, however, there are still many issues to take into account when designing mobile maps. It is important to consider how the user will interact with the application. If it is too complex, the user will have a difficult time completing tasks. Resolution and orientation of the display are also important factors due to the limitations of pixel and screen size. One of the greatest concerns when constructing mobile maps is what layers are needed and how to display all of the information. According to ESRI, "most mobile maps are composed of two primary types of layers basemap layers and operational layers" (ESRI, 2015). Because mobile devices have limited computing speeds, the choice of layers and basemaps are very important to the overall functionality of the web application.

For a project on Web-GIS Services for Sustainable Ecotourism, Chisa Nishii (2007) created two mobile web applications for collecting and displaying ecotourism data. The first website was simple and met the client's requirements, but it was a rudimentary design with limited functionality. The second map, however, included many functionalities that were more helpful to potential tourists than the customer by providing more detailed information about the specific areas. Although it contained more information regarding local tourist areas and allowed the audience to interact with the map, it also required more development time, and the application was slower than the 
first. One conclusion is that it might be best to create a map that is somewhere between simple and complex, creating a map that satisfies all of the audience and client's needs, but does not require intensive programming and design techniques.

Web GIS is also a valuable tool for collecting and disseminating data. In 2008, Geraldo Torres-Ruiz completed a study on disseminating geodetic data of military airfields through a web-based GIS application. Torres-Ruiz sought to provide an easier way for the National Geospatial-Intelligence Agency (NGA) to share data related to military airfields, including the location of runways, obstructions, and other points of interest to their clients. Once the data had been checked for accuracy, they were hosted on a server to be used in the application. In the past, NGA clients needed to make a request and wait for a NGA employee to compile the data, but with the use of the application, clients can now query and build the requests for themselves based on the data provided in the application. Torres-Ruiz concluded that the application not only made the dissemination process faster for the NGA, but also "provided its clients with analytical tools to explore the available data" (Torres-Ruiz, 2008, p.76).

\subsection{Planning and Modeling with GIS}

With the advancements of information technology over the last decade, many planning professionals are turning to GIS to visualize and communicate their models. Professionals such as urban planners and transportation engineers can accurately model a detailed, real world representation of the problem they are trying to solve. Modeling with GIS can help predict future environmental, developmental, and land-use patterns (Brail and Klosterman, 2001). 
A 2013 report from Shreyas Shinde explored the use of GIS for modeling human movement over terrain using nodal and vector analysis. By identifying "bottlenecks on a terrain," (Shinde, 2013, p.1) route analysis can be used to plan the optimal path for human movement through the terrain. This method can be used for planning search and rescue missions in a remote wilderness environment or military research of infantry movement (Shinde, 2013). With this least cost method, planners can find the most efficient route through the environment. Visualizing the data this way also allows the user to see the whole picture rather than just the simple solution.

The study in 2009 by David Robinson looked to help the International Civil Aviation Organization (ICAO) in modeling civilian airfields by setting up a GIS framework for organizing the data (Robinson, 2009). The study created a standard for modeling digital terrain and obstacle data, allowing the organization to share airport data with pilots. By modeling an airport's surroundings, pilots can now plan and analyze the safest approach routes to airfields around the world by analyzing data in specific coverage areas.

"We live in a 3D world. It makes sense, then, that people would find it easier to understand and explain the world using 3D models" (Fu, 2015, p.355). One of the main advantages of modern digital mapping is the ability to map or model environments three dimensionally. It enables the user to visualize the data in a more realistic way that can be better interpreted for analysis. At the 2006 Esri User Conference, Steve Ramroop created a 3D model of Troy University in Alabama. According to Ramroop, "The common 3D model is a comprehensive land-related information system which includes land use, buildings, flood hazards, population at parcel level" (Ramroop, 2006). During the process 
of creating the 3D model, Ramroop used contour lines to generate a Triangulated Irregular Network (TIN) model. A Digital Elevation Model (DEM) was then created using the TIN and a georeferenced aerial photo was draped over the DEM. This created a 3D model of the terrain, showing the elevation changes throughout the environment. Buildings were also digitized in a 3D format so that they could be displayed with height extrusion values.

Donna Knox (2004) from the University of Southern Queensland took 3D terrain modeling to another level when she created a virtual fly-through for her map on tourism conservation. Knox utilized the same process Ramroop used to create a DEM and 3D model of terrain. However, she then used ArcScene to create a compiled sequence of events from the map which, when played in order, gave the effect of flying through the environment. Although Knox succeeded in creating the animation, she claimed that there were several limitations, including memory problems and that "export time for one video animation was 30 minutes" (Knox, 2004, p.95). DEM file size and data recall abilities are some of the biggest limiting factors when working with 3D animations.

\subsection{Summary}

Technological advances in the web and mobile communities have led to an increase in GIS technologies being implemented over the web. By hosting data and GIS applications over the web, more end users have the ability to access and analyze the data. Mobile capabilities also allow users to access these web applications on mobile devices. However, in order to have a responsive application, the data used should be simplified and implemented based on necessity. Planning and modeling using GIS technologies has vastly improved analysis and predictive results. GIS software allows for better 
visualization of real world entities. 3D modeling can also be used to add another dimension of data analysis for the user. They allow the user to explore the scene in a more lifelike representation. 


\section{Chapter 3 - Systems Analysis and Design}

Before starting work on a new project, an exploration of the system design and project requirements is essential in order to guarantee all of the goals of the project are met at the end of the project. By doing this, a project plan can be built that takes all aspects of requirements of the project into consideration. In this chapter, an exploration of the Thunderbirds Routine Mapping Application project plan will begin by revisiting the problem statement in section 3.1 and continue with an analysis of all client requirements in section 3.2. A discussion of the system design and project plan are in sections 3.3 and 3.4 respectively.

\subsection{Problem Statement}

The paper map system that the pilots had been using for planning their routines at various locations had become too cumbersome. In addition to time constraints, the pilots faced problems with visualizing the entire routine on one paper map and were unable to quickly edit the map if a potential hazard was found or a point location needed to be changed.

\subsection{Requirements Analysis}

In order to obtain a full understanding of the client's expected final product, it was important to analyze the requirements the client set forth for the project. Doing this ensured all the client's needs were met and helped to prevent delays. Two different types of requirements were present in this project: functional and non-functional.

Functional requirements explain the capabilities that a system is expected to provide. These requirements can range from simple processes like "zoom in/out" to more complex functions and processing, but they all explain what the product must do. Non-functional 
requirements, on the other hand, explain how the system must operate or perform when accessed by the user. These requirements are more focused on usability, operational time frames, and interfaces. Table 3-1 shows a list of all the functional (F) and non-functional (NF) requirements associated with the Thunderbirds Mobile Web Application. 
Table 3-1: Thunderbirds Routine Mapping Application Requirements

\begin{tabular}{|c|c|c|}
\hline Type & Requirement & Description \\
\hline $\mathrm{F}$ & $\begin{array}{l}\text { Accept user input for show } \\
\text { center and Bomb Burst } \\
\text { point }\end{array}$ & $\begin{array}{l}\text { Application accepts user input coordinates in } \\
\text { degree decimal minute format for two important } \\
\text { air show locations }\end{array}$ \\
\hline $\mathrm{F}$ & $\begin{array}{l}\text { Flight routine points } \\
\text { dataset auto-generation }\end{array}$ & $\begin{array}{l}\text { Based on the accepted user inputs, the } \\
\text { application calculates and creates the locations } \\
\text { of the points for the entire airshow }\end{array}$ \\
\hline $\mathrm{F}$ & $\begin{array}{l}\text { Display potential aerial } \\
\text { obstructions from } \\
\text { buildings }\end{array}$ & $\begin{array}{l}\text { Application displays the location of all buildings } \\
\text { in the show area that could potentially obstruct } \\
\text { the flight path of the jets }\end{array}$ \\
\hline $\mathrm{F}$ & $\begin{array}{l}\text { Calculate true heading and } \\
\text { flight angles }\end{array}$ & $\begin{array}{l}\text { Application calculates and displays magnetic } \\
\text { heading and flight angles necessary for the pilots } \\
\text { to navigate the airshow }\end{array}$ \\
\hline $\mathrm{F}$ & Export Map & $\begin{array}{l}\text { Application provides functionality to export the } \\
\text { map to a JPEG image }\end{array}$ \\
\hline $\mathrm{F}$ & Login & $\begin{array}{l}\text { Application requires user to login using proper } \\
\text { credentials }\end{array}$ \\
\hline $\mathrm{F}$ & 3D Visualization & $\begin{array}{l}\text { Application allows users to view the airshow in a } \\
\text { 3D format }\end{array}$ \\
\hline NF & Quick map creation & $\begin{array}{l}\text { Application produces a final map in less than one } \\
\text { hour }\end{array}$ \\
\hline NF & Multiple platform & $\begin{array}{l}\text { Application works on phones, tablets, laptops, } \\
\text { and desktops }\end{array}$ \\
\hline NF & Graphic User Interface & $\begin{array}{l}\text { Application interface is arranged in a simple } \\
\text { layout }\end{array}$ \\
\hline NF & Open Source & Project must use Open Source software \\
\hline
\end{tabular}




\subsection{System Design}

After the requirements of the final product were analyzed, a system design was created to map out and show how the different parts of the system would interact and function together. Figure 3-1 shows the system design for this web application.

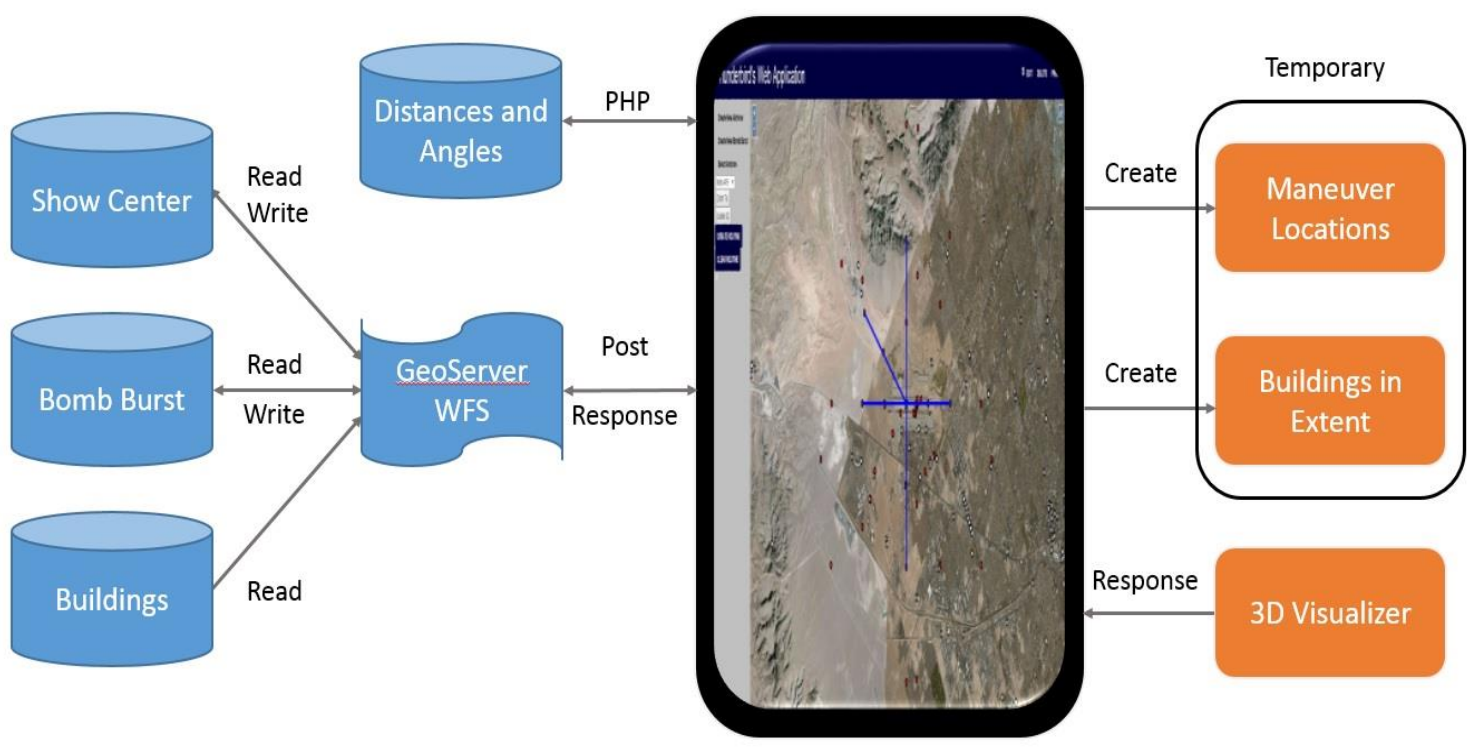

Figure 3-1: System Design

A single database, containing the geographic information for the application, was used in the project. This database holds four different datasets: Show Center, Bomb Burst, Distances and Angles, and Buildings. These datasets were then hosted using GeoServer Web Feature Service (WFS) protocols with read and write capabilities except the Buildings dataset, which was only given read capabilities. The Distances and Angles dataset was accessed through a PHP script. The application communicates with the server to access these datasets through feature service request posts and responses. Application users can access the system through their mobile devices and desktops. A valid web browser was required to access, as are proper login credentials in the form of a military 
issued Common Access Card (CAC) for desktops and a username/password combination for mobile devices.

Using the application, the user has the ability to create new airshows and view, edit, or delete old ones. The application accepts user input for the location of the Show Center and Bomb Burst locations for each new show. These points are easy to edit and delete through functionality offered in the application. This information is passed back to the server through a transaction post on the WFS, which updates and saves the database. After selecting which Show Center and Bomb Burst Location to use, the user can create a new airshow routine map. The application calculates the locations of all the maneuver points and creates a temporary layer containing their geometries. A second temporary layer is created that contains all of the buildings within the extent of the airshow. The data layers in the 2D map can also be visualized in a 3D environment that is provided in the application. The conversion is completed automatically when changing the map scene.

\subsection{Project Plan}

A project plan was also established at the beginning of this project and provided a framework for project completion. In order to better organize the project, three phases were used for dividing the tasks needed to complete the Thunderbirds Routine Mapping Application: design, development, and deployment (Figure 3-2). The final deliverables were also taken into account in order to make sure all aspect of the project were being accounted for. 
Design

- Requirements

- Data Collection

- Models
Development

- Data Cleanup

- Database

- Application

\section{Deployment}

- Testing

- Approval

- Migration

\section{Figure 3-2: Project Plan Phases}

\subsubsection{Design Phase}

During the design phase, most of the project plan was laid out to be completed in multiple tasks. The first task involved speaking with the client and the U.S. Air Force Thunderbirds commanding officer to come to an agreement on the scope of the project. This included identifying the client's problem and all requirements for the final product. The second task was a discussion about the data needed to satisfy all of the requirements for the project. During this task, research was also completed on the availability of the required datasets and at what geographic extent, as national level data were preferred. Task three focused on the creation of a logical and conceptual model (see Chapter 4) in order to see how the airshow is represented in the real-world environment and how it should be represented in the database. The final task for the design phase was to identify the methodology and software needed to produce the final product. During this phase, it 
was decided that the open source software suite OpenGeo would be used for all aspects of the project.

\subsubsection{Development Phase}

The development phase included the creation of all the databases, processes, and application scripting, which involved a considerable amount of testing since each task needed to function properly before the next task could begin. The first task of this phase was to create the database and datasets containing the necessary geographic information. Before the data could be hosted in a dataset, some preprocessing was completed to meet database and application standards. The datasets were then created using PostGIS and hosted on GeoServer as WFSs. The next task was to script the base web application using a combination of JavaScript, HTML5 and CSS. The application layout was scripted using Bootstrap CSS, while the application functionality was integrated using the JavaScript API. The next task involved creating and adding the various functionality to the application. A JavaScript function was scripted that calculates the coordinates for the different maneuvers in each airshow. This step also included the implementation of the application tools including zooming in/out, rotating north arrow button, accepting user input from forms, calculating true headings of the jet flight paths, exporting maps to an image file, and the ability to create, edit and delete airshow locations. The last task in the development phase was to add a 3D visualizer, which was created using OpenLayers3's Cesium extension. This task also included adding 3D building data for the aerial obstructions dataset as Cesium primitives and a layer containing 50 meter buffers around each airshow maneuver point. 


\subsubsection{Deployment Phase}

The final phase of the project was to deploy the application. While testing was completed after each task in the development phase, testing was also completed in this phase in order to ensure that the application worked on multiple mobile devices and web browsers. After all testing was completed, the application was submitted to the client for approval. Upon receiving feedback, final edits were made and the final application was submitted to the client. All associated data, files, and scripts were also transferred over to the client.

\subsubsection{Deliverables}

There were three deliverables associated with this project. The first deliverable, the data, was hosted on the client's GeoServer account, so there was no data migration needed as the MDEP already had access and full control of the database. The application was the second deliverable. For this, the client received the JavaScript, HTML5, PHP, and CSS script files used for creating the application. The final deliverable was a user guide that explained the use cases and the functionality implemented in the application. This user guide was provided to the Thunderbirds as a training guide for the pilots and staff.

\subsection{Summary}

After exploring the client's requirements for the Thunderbirds Routine Mapping Application, a project plan was created that subdivided the project into three distinct phases. The Design phase was used to complete all research and layout the project plan. During the Development phase, the data were scrubbed and hosted on the server. The application was developed using two JavaScript libraries during this phase. The application and all associated data, files, and scripts were then transferred to the client 
during the Deployment phase. MDEP then hosted the application on its server to be used by the Thunderbirds. 



\section{Chapter 4 - Database Design}

One of the most important aspects of a geographic information product is the data used and how they are stored and implemented in the system. By storing the necessary information in a database, the system can easily query and access data while staying organized. This chapter explores the database structure used while building the Thunderbirds Routine Mapping Application. Section 4.1 discusses the conceptual model used for the project; Section 4.2 explores the logical model used for implementation purposes, and data sources; and data migration are discussed in Section 4.3. Section 4.4 gives a summary of the chapter.

\subsection{Conceptual Data Model}

In order to fully analyze and break down how the objects interact and relate to each other in a real world context, a conceptual model was created (Figure 4-1). A conceptual model helps by graphically displaying the layout and environment of the entire situation, which allows for a better understanding of how everything works together. In this project, the problem focused around creating airshow maps for the Thunderbirds. To break the model down, each airshow is comprised of a number of maneuvers. One such maneuver - the bomb burst - is essential for lining up and measuring the true headings of the airshow. In each airshow, there are a number of aircraft flying in the environment (airspace) while trying to complete the maneuvers. At any time during the airshow, there could be a range of one to four aircraft in the environment at a time. There are potentially buildings that are also located in the environment that the aircraft must avoid. 


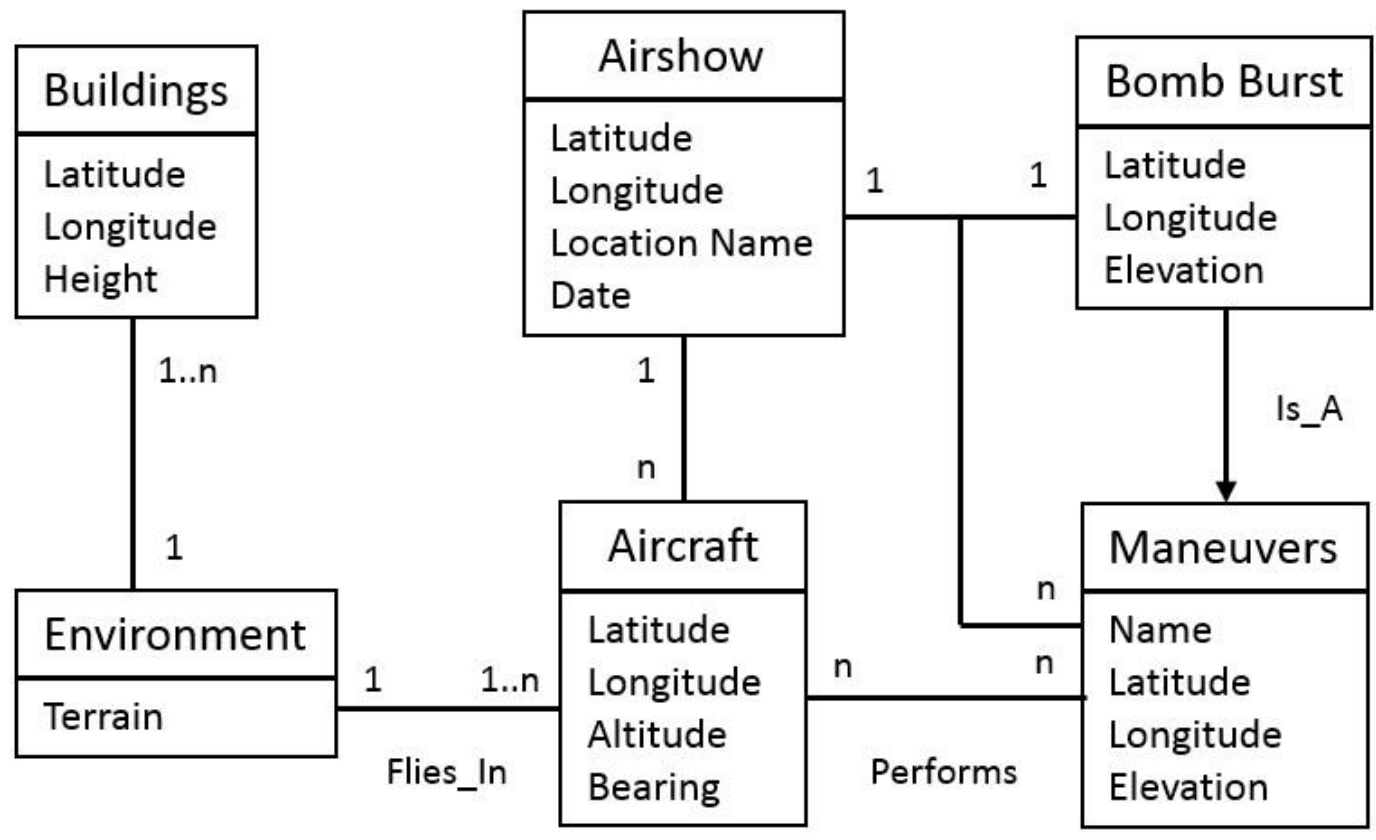

Figure 4-1: Conceptual Data Model

\subsection{Logical Data Model}

The logical data model shows how the datasets are organized and related in the project solution. Showing a flow of how the data are incorporated into the solution is beneficial for database structure and for programming the web application. Figure 4-2 shows the logical data model that was used for developing this project. All entities from the conceptual model have been included, but have been represented and accessed in different ways. 


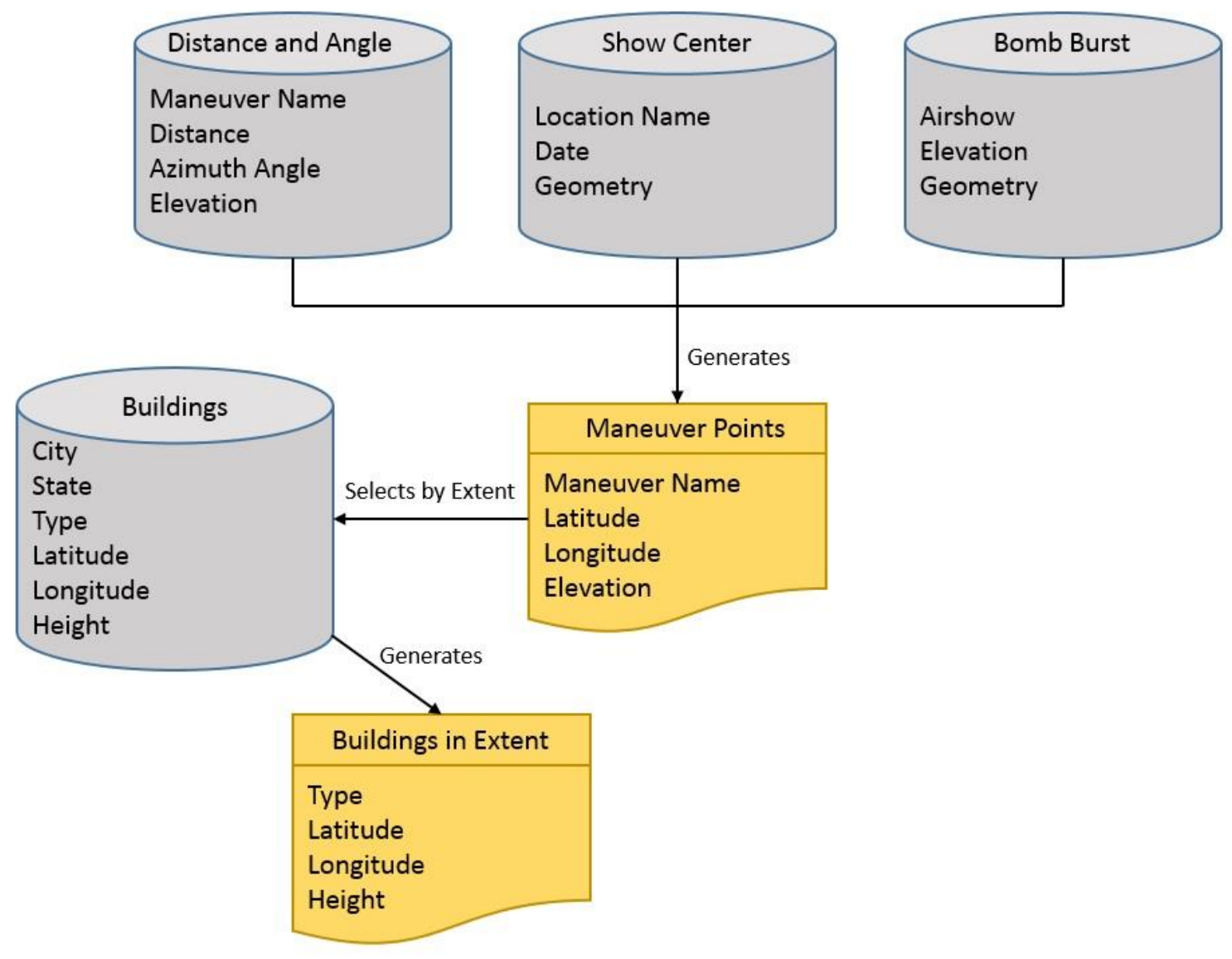

\section{Figure 4-2: Logical Data Model}

Four datasets were used in the implementation of the Thunderbirds Routine Mapping Application. Since the aircraft are in constant motion during the airshow, their location in the application is represented by the locations of the point maneuvers. These maneuver points are created in a temporary layer using the "Distance and Bearing," "Show Center," and "Bomb Burst" datasets. The datasets for the center location and the "Bomb Burst" are created using user input for location information, while the maneuvers points are calculated in the application. Airshow locations are represented by the "show center" points. After a show routine is calculated, the extent of the maneuver points is used to select the buildings in the area. These buildings are then imported into the application as $2 \mathrm{D}$ points and Cesium primitive objects to allow for height extrusion in the 
3D visualization. All of the datasets used in this project were hosted in a database in PostgreSQL. The show center and "Bomb Burst" datasets are related based upon their "Location Name" and "Airshow" fields.

\subsection{Data Sources, Scrubbing, and Migration}

Only three data sources were used to gather all of the information needed for the project. Using reliable data sources is paramount in any project, as inaccurate, incomplete, or false data render any results invalid. All of the data received also had to be formatted correctly before being imported into the system. The following sources were used for data collection.

\subsubsection{Maneuver Locations}

The airshow routine data used for this project were provided by the Thunderbirds in three different formats. A Google Earth Keyhole Markup Language (KML) file contained the routine points for a sample airshow at Nellis Air Force Base (AFB). Using this file, the geographic coordinates of each point were used to calculate the distance between each point and the airshow center location and the azimuth from the center to each routine point. A Python tool was scripted in order to calculate these values, which were then stored in a dataset and incorporated into the application to calculate the location of the maneuver points for each new show.

In addition to the KML file, a PowerPoint Presentation file which contained the directions for how the points were created in Google Earth was provided. This file contained the elevation data needed for the application and also provided some distances and angles between maneuver points and show center. These distances and angles were used to check the accuracy of the information calculated by the Python tool. The file did 
not contain complete location information for all flight maneuvers, so the two files were used together to check and calculate the locations. Finally, two additional sample Thunderbird routine maps were provided as a means to visually confirm the accuracy of the created maps and to show how airshow routines should be displayed. These maps were released by the Air Force as Unclassified/Limited Distribution and were produced by the National Geospatial-Intelligence Agency (NGA).

\subsubsection{Building Obstruction Data}

The dataset containing the location of all buildings that could be potential aerial obstructions was obtained as a collection of Digital Obstacle Files (DOF) from the Federal Aviation Administration's (FAA) Aeronautical Information Management service. There is one ASCII file for each state and some U.S. territories, and it is updated every 56 days for all new verified and un-verified man-made structures for public consumption. For this project, it is assumed the information provided by the FAA is up to date and accurate. Each file has the center point location information, above ground height (feet), and above mean sea level height (feet) for every building in the state, using the World Geodetic System 1984 (WGS 84) datum and the North American Vertical Datum of 1988 (NAVD 88). Before being converted to a PostgreSQL database, the location data had to be edited and formatted in a Microsoft Excel document to remove any unnecessary characters.

The location information contained in these files also had to be converted from degrees, minute, second coordinates to decimal degrees in order to be correctly plotted by the application. The location information contained inconsistent formatting and letters in numeric fields. New fields were created to contain the same information in the correct 
format. Negative signs were also added to longitudes that were measured in degrees west. The coordinates, in degree minute second format, then needed to be converted to decimal degrees. This was done by dividing the minute column by 60 , the second column by 3,600 , and then adding (or subtracting if in a negative longitude) the degrees, minutes, and seconds together.

\subsubsection{Terrain Provider}

The terrain web service used in the 3D visualization layer was comprised of different elevation data sources compiled and hosted by Analytical Graphics, Inc. (AGI), the official terrain provider for Cesium. On a global scale, the terrain model has a maximum spacing of 1,000 meters between height samples, but in the United States the maximum spacing ranges from 3 to 30 meters. An overview of all dataset resolutions is presented in Figure 4-3.

\begin{tabular}{|lll|}
\hline \multicolumn{1}{|c|}{ Source } & \multicolumn{1}{c}{ Coverage } & Approximate Maximum Spacing Between Height \\
Samples \\
$\begin{array}{l}\text { National Elevation } \\
\text { Dataset (NED) }\end{array}$ & United States & 3 meters to 30 meters \\
\hline EU-DEM & Europe & 30 meters \\
\hline $\begin{array}{l}\text { Australia } \\
\text { SRTM-derived 1 } \\
\text { Second DEM }\end{array}$ & Australia & 30 meters \\
\hline CGIAR SRTM & $\begin{array}{l}\text { Approximately }-60 \text { to } 60 \text { degrees } \\
\text { latitude }\end{array}$ & 90 meters \\
\hline GTOPO30 & Entire Earth & 1000 meters \\
\hline
\end{tabular}

\section{Figure 4-3: Terrain Height Sample Spacing}




\subsection{Summary}

In this chapter, conceptual and logical models were analyzed and compared. Due to the constantly changing location of the aircraft, multiple datasets were used as a means of representing their location. Using location information from the aircraft and buildings, combined with 3D terrain visualization, potential aerial hazards can be analyzed with the application. 



\section{Chapter 5 - Implementation}

After setting up the system design and gathering all the data, the next phase of the project was to create the web application. As mentioned in Chapter 1, this project relied on the use of the open source software suite OpenGeo for database creation, data hosting, and application development. With the exception of a few smaller extensions, OpenGeo is a web-based software package that is accessed through a server. The Mojave Desert Ecosystem Program (MDEP) was responsible for building and installing the OpenGeo server used in this project. In the following sections, the implementation steps taken for Database Development (Section 5.1), Hosting Data on the Web (Section 5.2), and Web Application Development (Section 5.3) are explored in further detail. For an exploration of how the data were scrubbed and migrated, see Section 4.3.

\subsection{Data and Database Development}

The databases used for this project were created using PostgreSQL with the PostGIS 2 extension installed. The PostGIS 2 extension allows for spatially enabled data like geometry to be added to the objection-relational database system that PostgreSQL provides. With the help of the desktop software pgAdmin, a connection can be made to specific PostGIS servers allowing for easier database creation and management of datasets through standard query language (SQL) expressions. All data created using PostgreSQL were stored on the server at the specified port. After a database was created and spatially enabled, it could be accessed by the different extensions in the OpenGeo suite. 


\subsubsection{Calculating Distances and Azimuths}

The flight routine points data used in the application were calculated from sample data before being implemented. Using the given KML file of a sample airshow, the coordinates of all the points in the sample routine were recorded and used to calculate the distance and azimuth angle (true heading) from the center location. Since the planes are flying in the air and not on a 2D surface, the great-circle distance (Figure 5-1) haversine calculation, equation 5-1, was used to calculate the distances:

$$
\begin{gathered}
\mathrm{a}=\sin ^{2}\left(\frac{\Delta \varphi}{2}\right)+\cos (\varphi 1) * \cos (\varphi 2) * \sin ^{2}\left(\frac{\Delta \lambda}{2}\right) \\
\mathrm{c}=2 * \operatorname{atan} 2(\sqrt{\mathrm{a}}, \sqrt{1-\mathrm{a}}) \\
\mathrm{d}=\mathrm{R} * \mathrm{c}
\end{gathered}
$$

Where $\Delta \varphi$ is change in latitude from $\varphi 1$ to $\varphi 2, \Delta \lambda$ is the change in longitude, $\mathrm{d}$ is the distance, and $\mathrm{R}$ is the Earth's mean radius.

The azimuth angle between the two points was calculated using equation 5-2:

$$
\begin{gathered}
\theta=\operatorname{atan} 2(\sin (\Delta \lambda) * \cos (\varphi 2), \cos (\varphi 1) * \sin (\varphi 2)-\sin (\varphi 1) * \cos (\varphi 2) \\
* \cos (\Delta \lambda))
\end{gathered}
$$

Where $\varphi$ is latitude, $\lambda$ is longitude, and $\theta$ is the azimuth angle. 

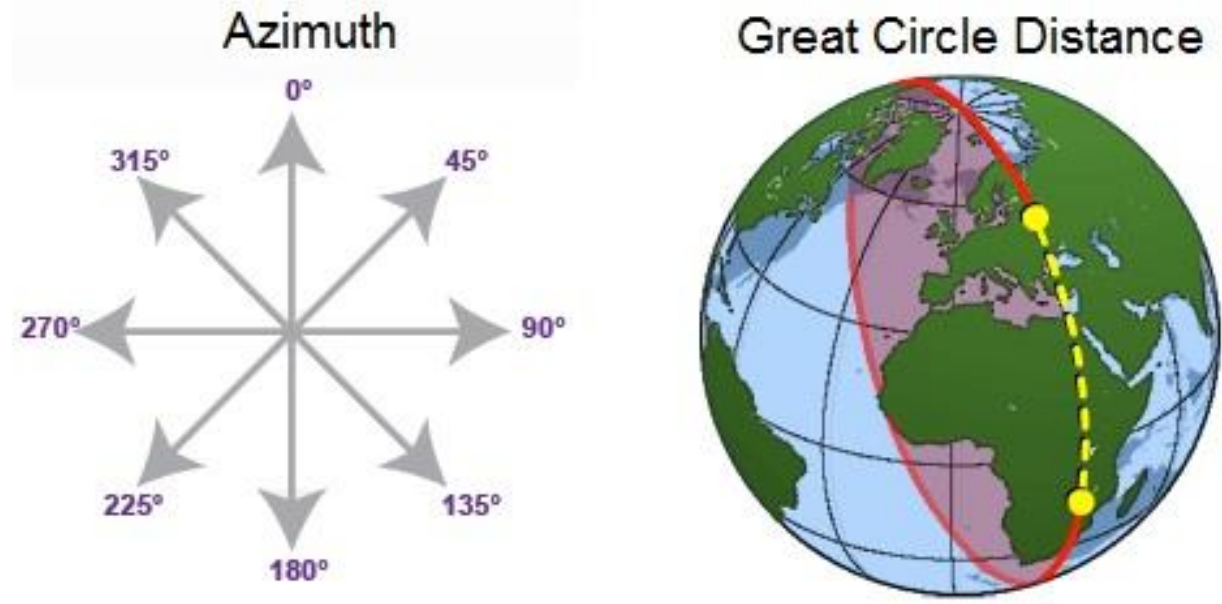

Figure 5-1: Azimuth and Great Circle Distance Representations

A Python script was written to calculate these distances and angles for each point in the routine. The Python script accepts user input for decimal degree coordinates, converts the coordinates to radians, and then calculates the distance and angle. The coordinates were converted to radians for use in the trigonometric functions in the equation. To convert to radians, the coordinates in degrees were multiplied by $p i / 180$. The distances and azimuths were then stored in a dataset to be used later when calculating the locations of the maneuvers for a new airshow.

\subsubsection{Creating a New Database}

The pgAdmin layout is split into two windows: an object browser (database tree) and a viewer window that allows the user to view the properties, tables, and statistics of the datasets. In the object browser, a new database can be created by right clicking on the server and selecting New Database. A popup window then allows for information needed in the database, such as projection and server connection URL, to be entered. However, this database is not yet spatially enabled. Using the provided SQL query button, a query window popup is used to spatially enable the database by creating an extension to 
PostGIS by typing and executing “CREATE EXTENSION postgis;”, which adds a spatial reference table to the database (Figure 5-2). This allows for a geometry field with a set projection to be added to the database, holding the geographic information data for each point. The projection is set in the dataset creation properties.

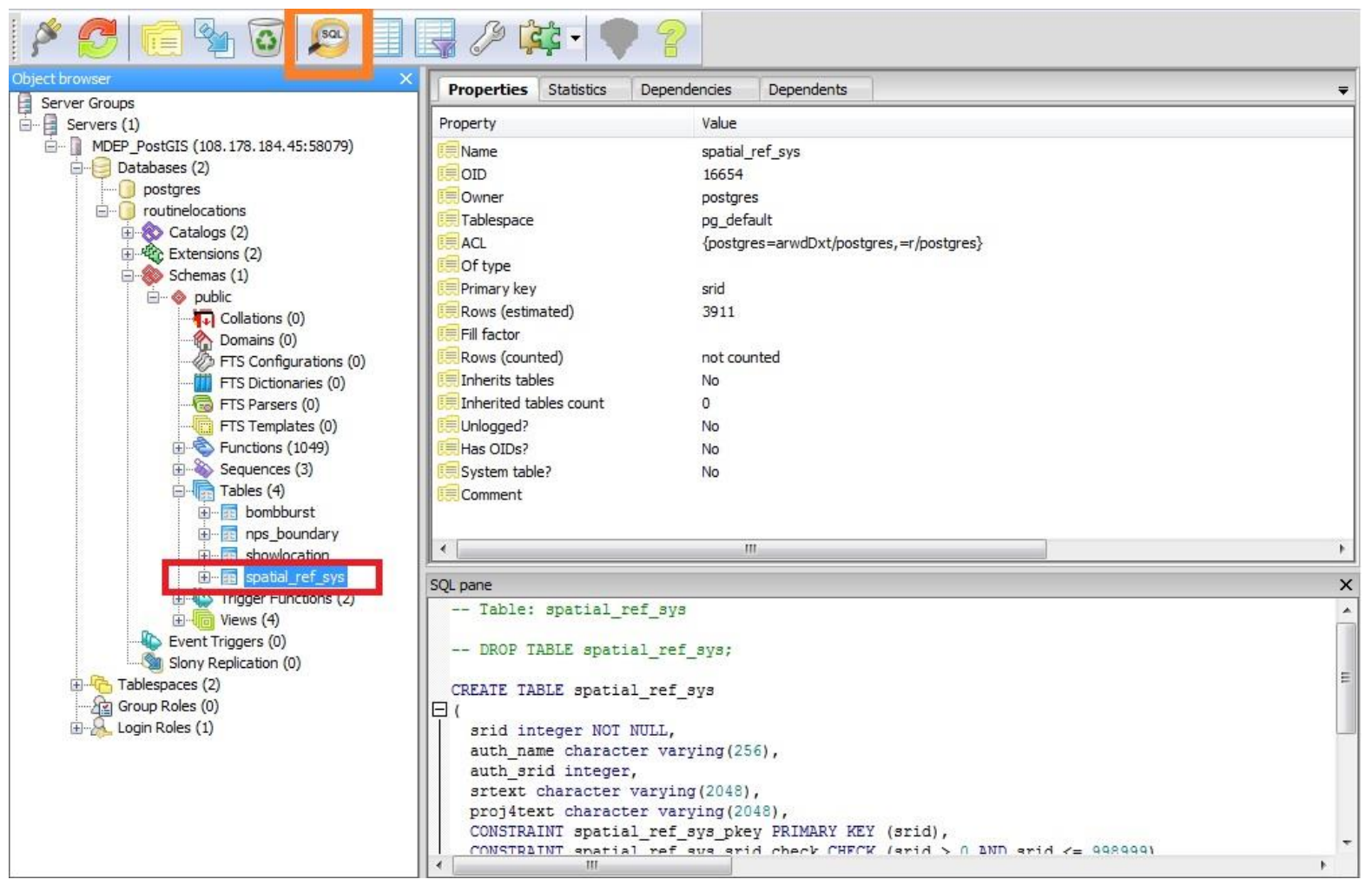

Figure 5-2: Database Layout in pgAdmin. The spatial_ref_sys table provides the spatial reference for the database. The $S Q L$ query button is boxed in orange.

\subsubsection{Creating Spatially Enabled Data Tables}

Once a database has been created, geospatially enabled tables (datasets) are created through a SQL statement. In this query, the table name and data fields are set as part of the "CREATE TABLE" function. Two tables were created for the Thunderbirds Routine Mapping Application: "Showlocation" and "Bombburst" datasets. A similar query can be used to insert data into the table (Figure 5-3). The following script was used to insert a data record into the "Showlocation" dataset used for this project: 
INSERT INTO showlocation (

geom,

location,

date)

VALUES (

ST GeomFromText ('POINT (-115.031667 36.2380)', 4326),

'Nēlis AFB',

' $\left.10 / 10 / 2015^{\prime}\right)$;

Figure 5-3: Sample Code for Inserting Data into a Table in PostgreSQL

The first half of this script defined which data fields the information will be inserted into and in what order. For the geometry field, a point type was established with the use of the "ST_GeomFromText()" function, which converts a string of coordinates at the specified location to the geometry type in the specified projection. The 4326 at the end of the geometry creation line represents the Spatial Reference System Identifier EPSG: 4326 (WGS84) and represents the projected coordinate system of the geometry. Changing this number changes the projection of the data. The next two lines specify the data that were inserted into the "location" and "date" fields.

\subsection{Hosting Data on the Web}

GeoServer was the OpenGeo extension used for this project that allowed for sharing and editing of geospatial data on the web. GeoServer offers a few different services that can be used to host the data: web mapping service (WMS), web feature service (WFS), web coverage service (WCS), and web processing service (WPS). WMS is the standard protocol for accessing georeferenced map images, such as raster files, on the server; WFS is used for accessing the actual geographic data (i.e. points, lines, and polygons) and their attributes; WCS is used for accessing the underlying raster coverage (base layer) data; and WPS is used for publishing geospatial processes and algorithms. Once a dataset has 
been hosted by GeoServer, it can be accessed through the web with these different services.

\subsubsection{Setting up the Workspace}

In order to host data with GeoServer, a workspace location must be set (Figure 5-4). In GeoServer, the user can create a "Workspace", "Store", and "Layer", preview a layer, and create/manage the layer styles. A "Workspace" acts like a folder and is created for holding all data relating to a specific project. A "Store" contains all data from a specific data source and type. When adding a new "Store", the type of file is selected based on the software used to create it. GeoServer supports various data formats, including Esri shapefiles, PostGIS databases, and raster sources. Stores can contain a single layer or multiple layers. For this application, a Thunderbirds workspace was created with two different data "Stores".

\begin{tabular}{|c|c|c|c|c|c|c|}
\hline 口 & Type & Workspace & Store & Layer Name & Enabled? & Native SRS \\
\hline$\square$ & 固 & opengeo & cntry_shp & countries & $\checkmark$ & EPSG:4326 \\
\hline$\square$ & 口 & usa & states_shp & states & $\checkmark$ & EPSG:4326 \\
\hline$\square$ & 。 & Thunderbirds & NevadaBuildings & Nevada & $\checkmark$ & EPSG:4326 \\
\hline$\square$ & - & Thunderbirds & routinelocations & showlocation & $\checkmark$ & EPSG:4326 \\
\hline$\square$ & e & Thunderbirds & routinelocations & bombburst & $\checkmark$ & EPSG:4326 \\
\hline
\end{tabular}

Figure 5-4: GeoServer Setup for the Thunderbirds Routine Mapping Application

\subsubsection{Adding Data Layers}

A "Layer" contains geospatial features that are of the same geometry (point, line, or polygon) and are the same type (i.e. trees, buildings, or streets). After the Thunderbirds workspace was created, a new store had to be added for each new source of data used. One was created for connecting to the PostgreSQL database and the other was created for connecting to an ArcGIS dataset. In GeoServer, creating a Layer prompts the user to 
select in which Store the dataset is located. Selecting a Store will then show all datasets that can be added and a new Layer must be created for each dataset used. The "Showlocation" and "Bombburst" datasets used in this project were hosted through the PostgreSQL database while the buildings dataset was hosted from the ArcGIS dataset.

Once a Layer is created in GeoServer, there are options for publishing the data for the different web services and setting up tiles for the dataset. Most of these options were left at default for the Routine Mapping Application, but the extent of the data layers was changed to fit the geographic scope of the project. The projection of the dataset was set and can be changed in these settings as well.

\subsection{Web Application Development}

The largest part of this project focused on developing the web application. The Thunderbirds Routine Mapping Application was built using JavaScript, HTML 5, CSS, and PHP. Scripting was completed and debugged in Notepad++ while the web functionality was debugged using Firefox Firebug toolkit. The application was geared more towards mobile device usage than desktop. However, the application can be accessed on both platforms. The open source JavaScript library OpenLayers 3 was also used in order to access the data hosted in GeoServer and provided most of the application functionality. Other functionality was produced using the Cesium extension API. PHP was used for basic querying of the PostgreSQL datasets.

The workflow in Figure 5-5 shows the layout of the functionality in the application. From the home page, the user can either create a new airshow or view an existing one. When creating a new airshow, the user enters locations for the show center location and the bomb burst location. A new point is then added to the database through a 
transaction function when the user clicks the map. If the user is viewing an airshow, the application asks for a show center and bomb burst location to be selected from dropdown menus. When the "create routine map" button is clicked, the application calculates the locations of the airshow points, airshow overlay, and selects all buildings in the obstructions database. The application also allows for the whole scene to be viewed in a 3D environment. In addition to the basic map used for the application, other functionality was added through the various methods explored in the following sections.

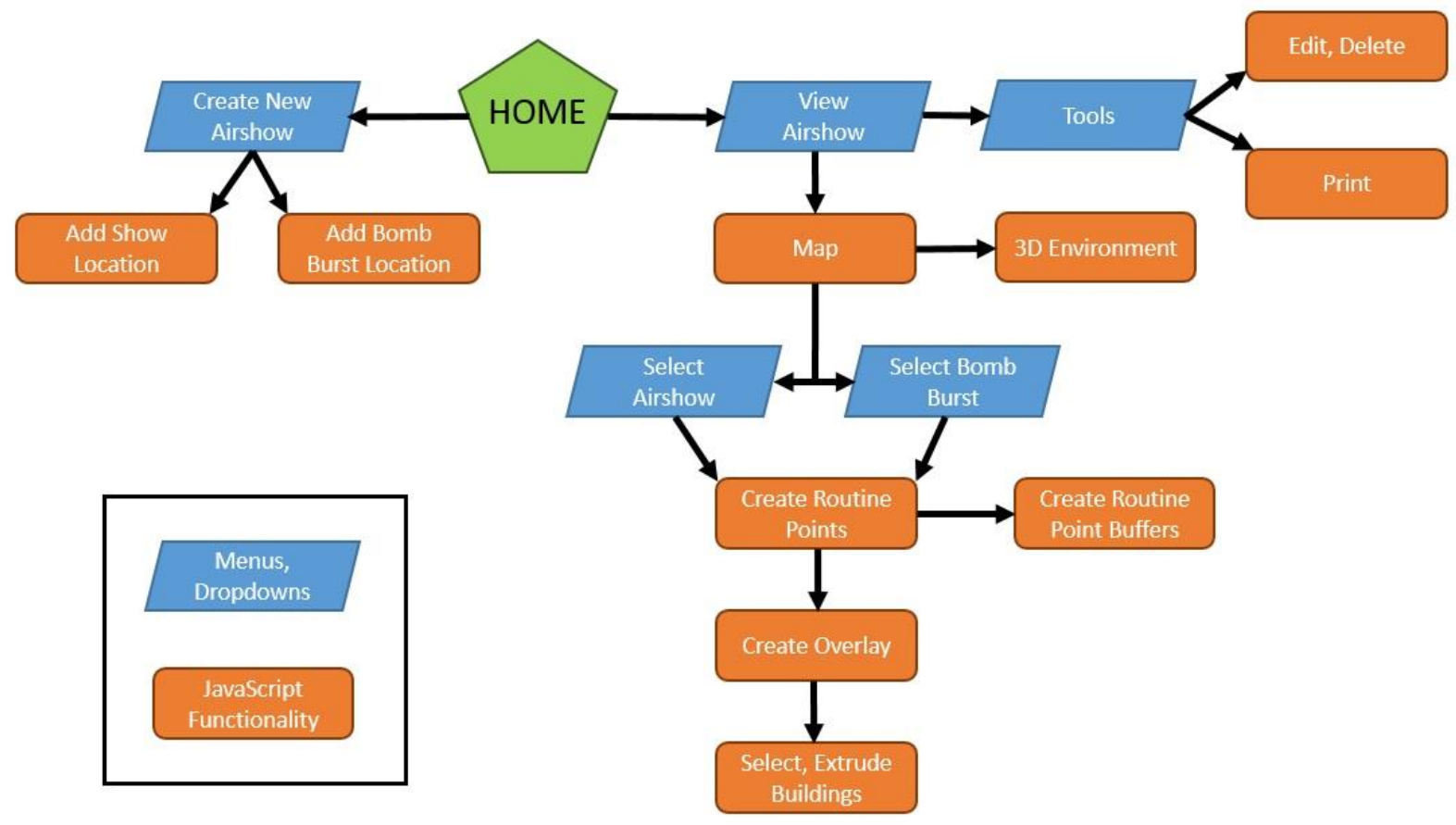

Figure 5-5: Thunderbirds Routine Mapping Application Workflow Model

\subsubsection{Web Map Creation}

The first part to creating the application was setting up the basic web map using the

OpenLayers 3 JavaScript library. In order to include functionality from the OpenLayers 3 library, the software was installed on the server and the respective CSS and JavaScript libraries were added as script source links at the beginning of the HTML file. After the 
libraries were included, a basic web map was added to the application with the "new ol.Map()" function. In this function, the different properties of the map were established, including the map controls, interactions, layers, and view. By default, the map included basic controls and interactions for zoom-in/zoom-out, but in the application the controls were extended to include touchscreen interactions.

For the Thunderbirds Routine Mapping Application, a base map layer hosted by Bing Maps was added (Figure 5-6). Bing Maps hosts different types of base maps, including the up-to-date satellite imagery used in this project, for registered users by providing a specific key number for each layer. The application also extends upon the defaults for controls and interactions by adding a North Arrow that rotates back to north when clicked. A "new ol.interaction.DragRotateAndZoom()" function was implemented to enable the application to allow the user to use their fingers for dragging, rotating, and zooming on the map in a mobile setting. This functionality can be simulated on a desktop web browser by using SHIFT + click. The map is automatically projected using the Web Mercator projection, so all datasets were reprojected using this projection. 


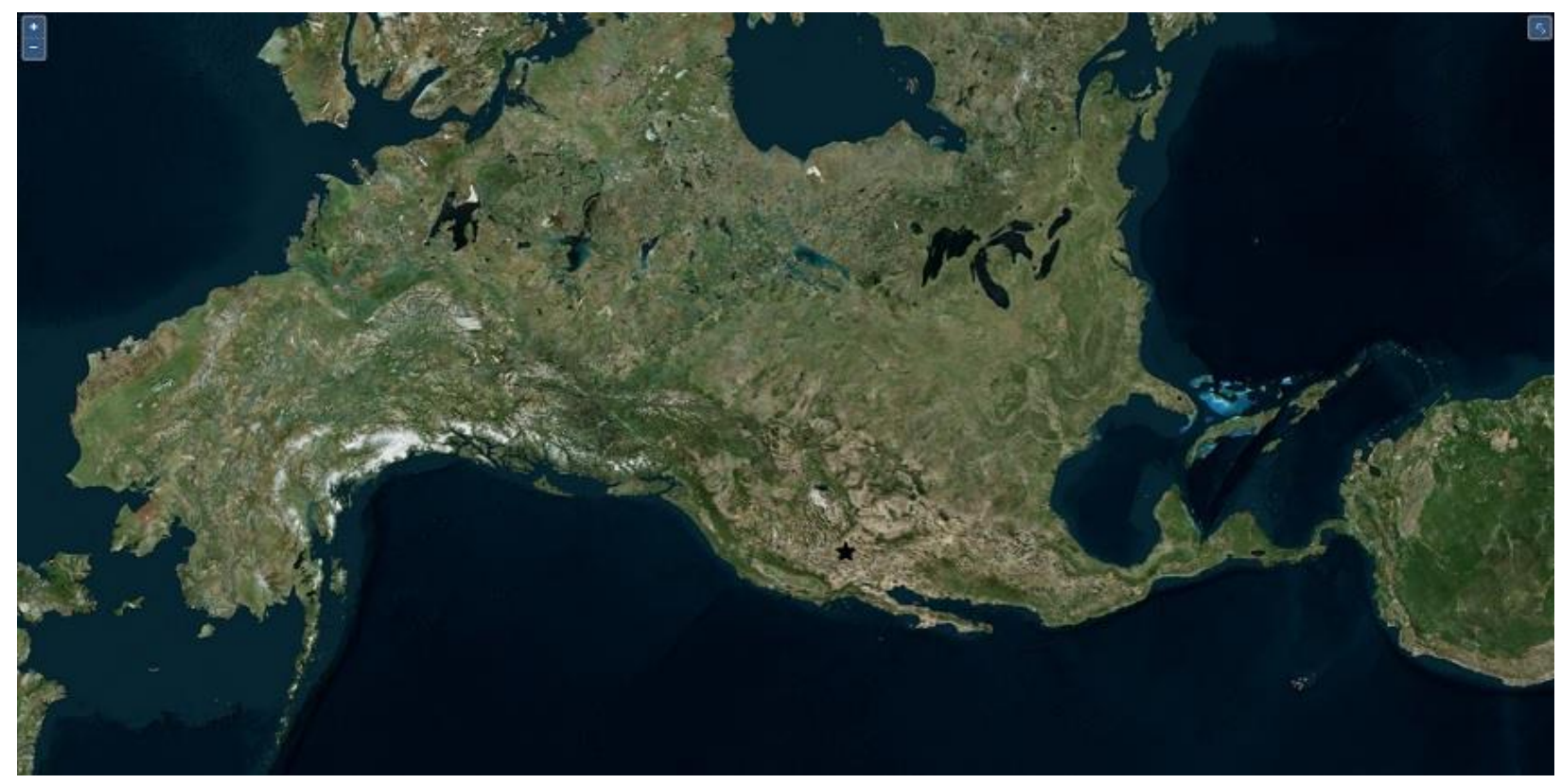

Figure 5-6: Basic Web Map with Bing Maps Satellite Imagery

\subsubsection{Adding Data Layers}

The OpenLayers 3 library provides Web Mapping Services (WMS), Web Feature Services (WFS), and Web Coverage Services (WCS) to request hosted data services from GeoServer. These services are based on Simple Object Access Protocol (SOAP), which works more efficiently with the object-relational database provided with PostgreSQL. The WFS protocol was used for this application because it allows for data creation and editing through a transaction request. For the application to access the data as a WFS, the source of the data need to be set using GeoJSON format and a JQuery loader. Both were provided with the OpenGeo suite. A different loader and format was used for each dataset in the application to avoid having the data loaded with the same symbology and styles.

When adding data as a WFS, the application sends out a series of requests to GeoServer using the link to the hosted service and bounding box (extent) of the data. The request is compiled automatically by the application based on the type of service being 
used. The data are pre-loaded as tile vectors to boost application loading speed. While the data are being loaded as a tiled vector, more requests are sent out depending on the tile settings in GeoServer. The application then reads the response and stores it using the GeoJSON format, which was then parsed to match the layer settings needed. The show center, bomb burst, and building datasets were accessed as WFS for this application. After the source of the data was set using the WFS functionality, the properties of the layer were also set, including the style, image type, and transparency. The point data used for this application were given a circle image type. Once a style was set, the layer could be displayed or added to the map. Figure 5-7 shows the style and symbology used in the application.

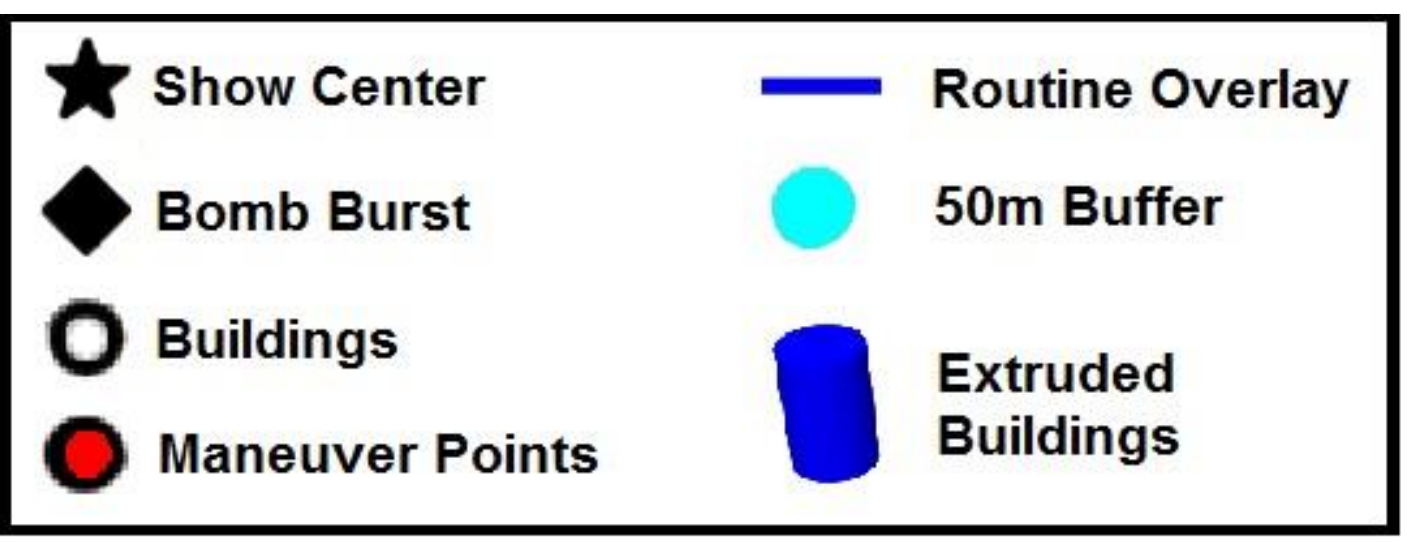

Figure 5-7: Symbology and Style for Data Layers

\subsubsection{Database Transactions}

As previously mentioned, WFS functionality was used with this application because of its ability to facilitate data creation and editing through a server connection. Using the script shown in Figure 5-8, information can be inserted, updated, or deleted based on the options used in the function. 


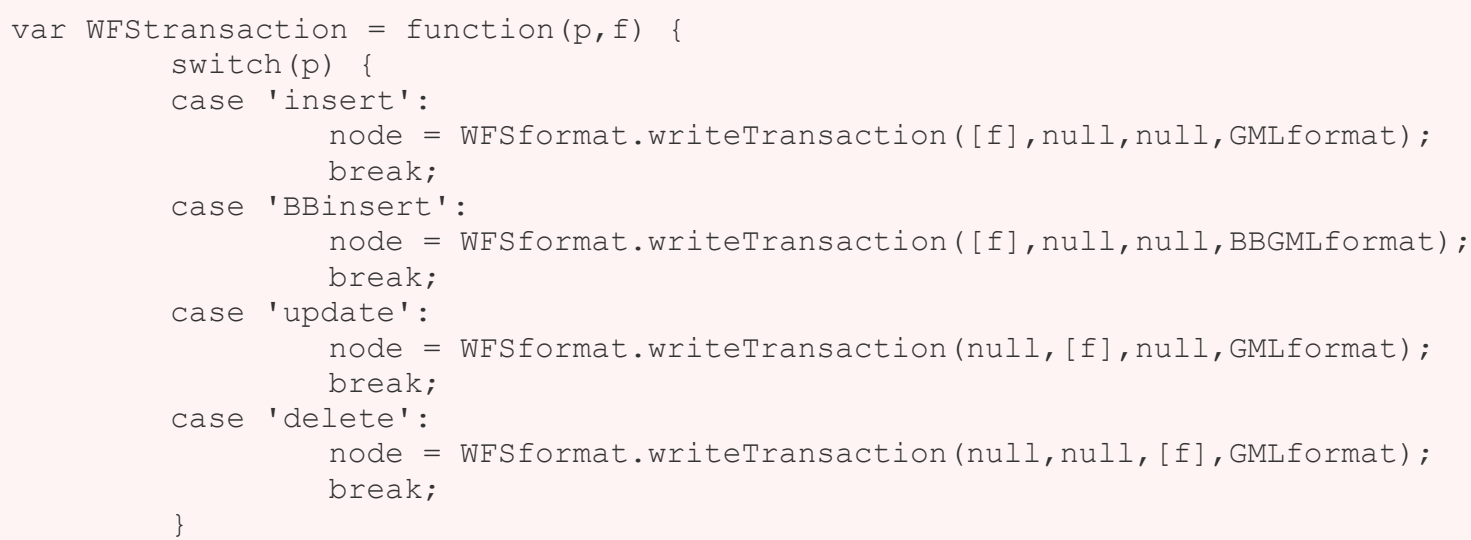

\section{Figure 5-8: Sample Code for Creating a Transaction Function}

In the application, the "writeTransaction()" function was used in conjunction with a select interaction that requests the feature information when the user clicks a point. This works for data already in the dataset, as well as for locations of new points. In order to add a new point, the interaction was used to draw a new geometry at the coordinates provided in the input forms. Since PostgreSQL is an object-relational database, and even though the point is drawn, it will not be saved to the database unless the geometry field in the database is set as well. The geometry field in each dataset contains the location and style information for each record. To accomplish this in the application, the geometry field was set in the JavaScript code. To edit the position of an existing point, the click interaction creates a clone of the feature at the new location and deletes the old feature. The geometry of the clone was set to match the new location and the FID was set to mimic that of the original. Deleting a feature was done by using the interaction to select a feature and deleting its FID, which erases the specific data record.

\subsubsection{Converting Input Coordinates}

To create a new airshow, users input coordinates for the center location of each new show. According to U.S. Air Force standards, the Thunderbirds report all coordinates in 
degree, decimal minute format. While this is acceptable for coordinate inputs, OpenLayers 3 and GeoServer do not recognize this format, so the coordinates needed to be converted to decimal degrees and then reprojected to match the map projection. To convert to decimal degrees from degree decimal minutes, the decimal minutes portion of the coordinate was divided by 60 and added to the degrees, giving one number in decimal degree format. However, if any of the coordinates are negative (such as the case with any longitude in the United States), then the decimal minutes need to be subtracted from the degrees. This was taken into account when programming the point conversion.

\subsubsection{Creating Routine Points}

The most important functionality for the application was the creation of the routine points. Not only does this show the location of each maneuver point, but it also sets up the layout for the flight headings overlay. The routine locations for each new airshow were calculated based on the show center location, bomb burst location, and their distances and azimuths. The azimuth from the show center to the bomb burst point is added to each routine point's azimuth stored in the database to handle the rotation of the routine layout and maneuver points relative to the show center. Figure 5-9 shows the workflow model for the maneuver point creation process.

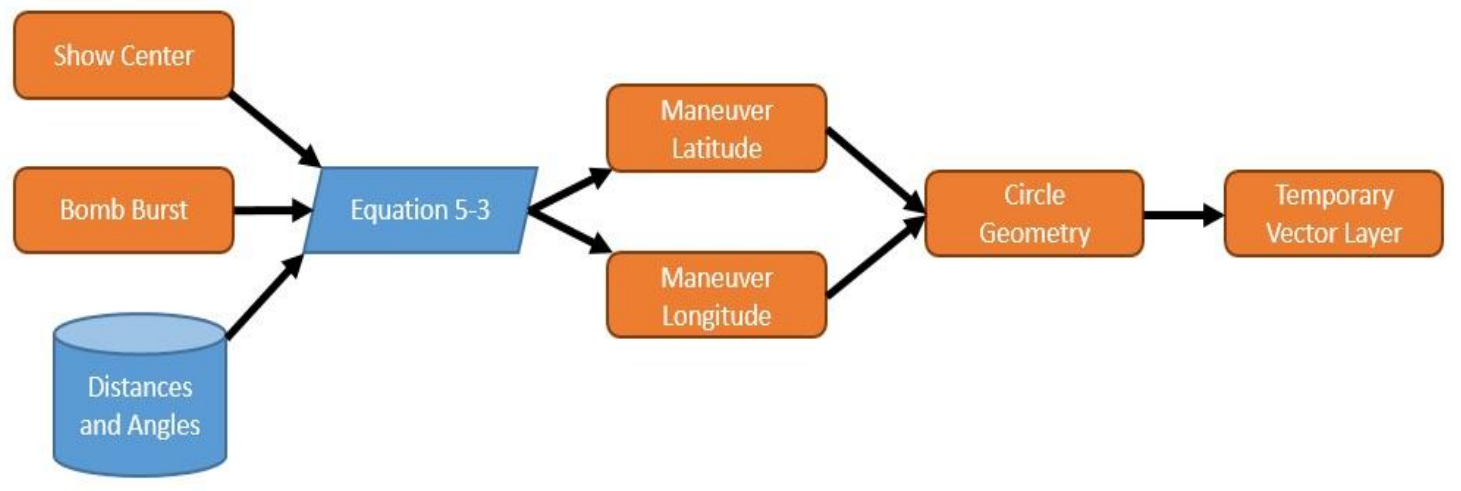

Figure 5-9: Maneuver Point Creation Process Model 
The azimuth and the corresponding distance for each maneuver were plugged into equation 5-3, solving for the longitudes and latitudes of each point for the new show.

$$
\begin{gathered}
\varphi 2=\operatorname{asin}(\sin (\varphi 1) * \cos (\delta)+\cos (\varphi 1) * \sin (\delta) * \cos (\theta)) \\
\lambda 2=\lambda 1+\operatorname{atan} 2(\sin (\theta) * \sin (\delta) * \cos (\varphi 1), \cos (\delta)-\sin (\varphi 1) * \sin (\varphi 2))
\end{gathered}
$$

Where $\varphi 1$ and $\lambda 1$ are the latitude and longitude of the show center location, $\theta$ is the azimuth angle, and $\delta$ is angular distance (distance/Earth's radius).

A JavaScript function was used to iterate through each record of the Distances and Angles dataset and calculate the coordinates for each maneuver point in the airshow. The coordinates were then passed into a "new ol.Feature()" function to create a circle geometry for the point. The geometries were then added to a temporary vector layer that was cleared after reloading the page or rerunning the "create routine points" function. The flight routine overlay, including all inbound and outbound headings needed by the pilots, was also created and added to the application using this process. The overlay consisted of three different lines with one going from the maneuver points 'show left' to 'show right', one from 'show back' to 'show front', and the other from the show center location to the maneuver point named ' 5 card perch'. To create the overlay, the coordinates for the stated maneuver points were used as end points of lines that were created to start from the show center. These lines were then also pushed to a temporary layer. The inbound and outbound true headings were also calculated and displayed with the routine overlay. Buttons were added to the application to allow the user to toggle layer visibility and to delete the temporary layers. 


\subsubsection{D Visualization}

To add some safety analysis aspects to the application, a 3D environment was incorporated that allows the user to look for potential hazards for the pilots. The 3D environment was implemented using the Cesium extension to OpenLayers 3. A separate JavaScript library for Cesium was included at the beginning of the HTML file in order to fully implement the viewer. By using the "new ol3d.OLCesium()" function, a new 3D map was enabled. By default, the 3D environment was flat and based on the World Geodetic System of 1984 (WGS84) ellipsoid by default, so a terrain provider was used to give the scene terrain elevation above the ellipsoid. The terrain model that provides 3D terrain visualization was provided by a Cesium partner group, but most terrain models can be used if given a link to the server location. The same touch functionality implemented for the 2D map also works for the 3D map with the added functionality that swiping up at the same time with two fingers changes the view angle of the map.

A temporary layer of buildings in the show extent was also added to the scene.

Using the location of the routine maneuvers, the buildings dataset, and the terrain model, the user can determine whether if there are any potential conflicts. The 3D scene is initially disabled in the application, but can be enabled with the "Enable 3D" button. Once the 3D environment was set up, the data added to the map were given 3D coordinates. For the Bomb Burst and maneuver location points, the height of each point was set by adding a z-value to the point creation geometry, which the Cesium environment automatically recognizes and uses in the scene. The terrain height was also sampled at each location and added to the z-value, giving a true height above ground value. The buildings in the application were brought into the $3 \mathrm{D}$ viewer, but in order for 
the buildings to be extruded, the layer had to be converted to 3D primitive objects.

Primitive objects are a representation of the geometry used in the $3 \mathrm{D}$ cesium scene. The terrain height was also sampled for each building so that the primitives would be on top of the terrain. Figure 5-10 depicts the process for how the buildings were added to the 3D scene in the Thunderbirds Routine Mapping Application.

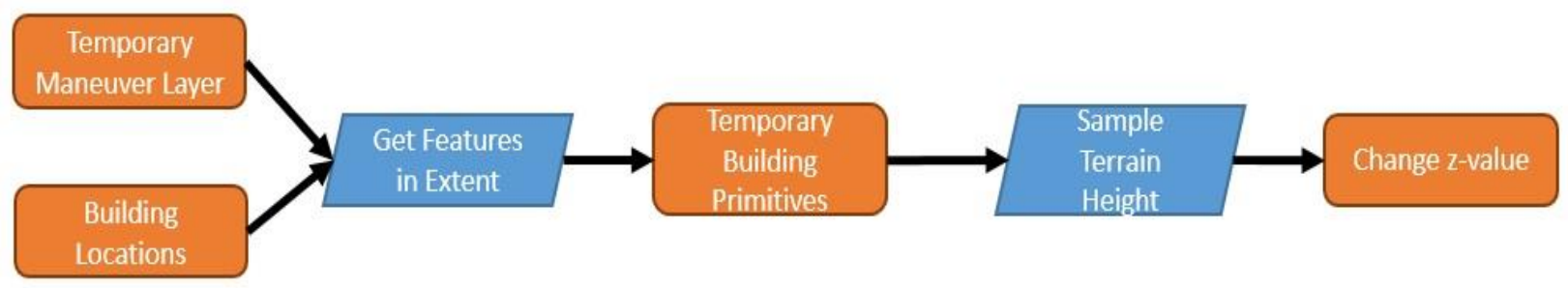

Figure 5-10: Model for Creating 3D Buildings in Airshow Extent

To select all buildings in the airshow, the "getFeaturesInExtent()" function was used to select all features within a given extent around the show center. The original buildings layer was removed from the map in order to benefit application loading speed. The coordinates queried for each building were used to create the new primitives layer, which was added to the map. This primitive feature class provided the ability to set extrusion dimensions for each building. Fifty-meter buffers were added to the 3D scene by creating a circle primitive at each maneuver point location using a similar process, and pushed to a temporary layer. The OpenLayers 3 API does not include geoprocessing tools, so the primitives were used to work around the lack of functionality offered in the library. Each layer can be individually turned on and off to optimize the 3D scene for analysis purposes using the check boxes in the layers dropdown menu. 


\subsection{Summary}

The Thunderbirds Routine Mapping Application relied heavily on JavaScripted functionality provided through the OpenLayers 3 library. Before the application could be implemented, a database was created using PostGIS 2 spatially enabled tables and then hosted on the server through GeoServer. Once on the server, the data were accessed through web feature service (WFS) protocol so that an "insert," "update," or "delete" transaction could be made. Temporary layers were created holding the routine maneuver points, point buffers, buildings in the show extent, and flight plan overlay, which were calculated through a scripted function. Finally, a 3D environment was added by utilizing the 3D terrain provider in the Cesium extension to OpenLayers 3. 



\section{Chapter 6 - Results and Analysis}

Once all of the data for the project have been hosted on the server, they can then be accessed and displayed in the JavaScript web application. The application was scripted to incorporate all functionality defined in the functional requirements of the project. This chapter explores the Thunderbirds Routine Mapping Application by discussing what functionality the application provides and how it is used. After the base application layout is explored in Section 6.1, instructions for adding a new airshow (Section 6.2), as well as creating and visualizing routine maps (Sections 6.3 and 6.4), are then explained.

\subsection{Application Layout}

Once the application is accessed through the web, the user is required to log in before use. If the user is loading the application on a desktop computer, he or she is asked to sign in via Common Access Card (CAC) information, or with a provided username/password combination. Mobile devices only allow for login using provided credentials because CAC readers cannot be implemented with any mobile device. After logging in, the application loads as seen in Figure 6-1. 


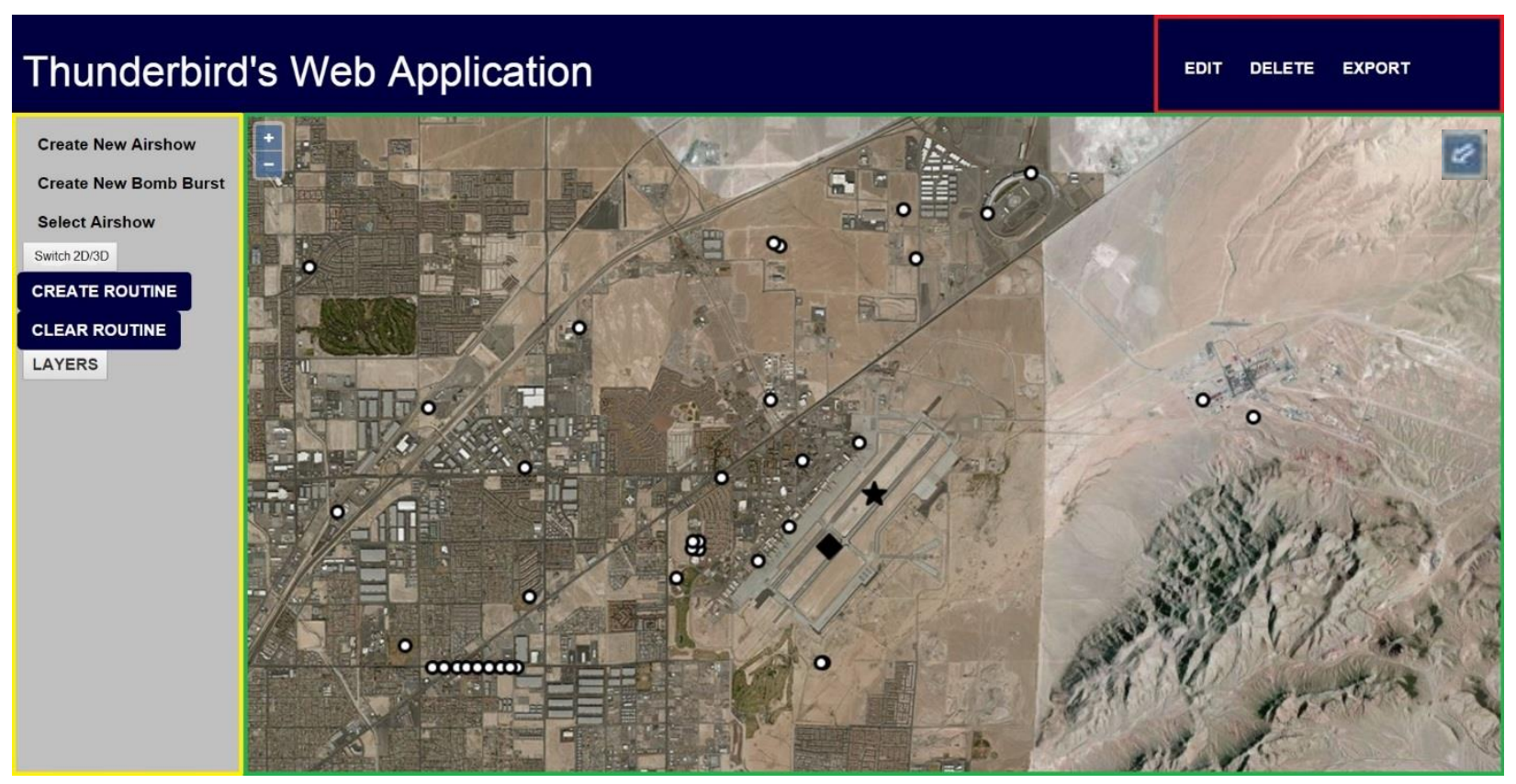

\section{Figure 6-1: Thunderbirds Routine Mapping Application Homepage}

The application is broken down into three sections: the map, map tools, and

routine tools. The map area (outlined with a green rectangle) is the largest part of the map and is used to display all data, layers, and the basemap for the application. The 3D visualization also populates this map area after the scene is enabled. Within the map pane, a user can zoom in and out with the "+,--" buttons on the left side of the map, or by double clicking the map, or by moving two fingers away from each other on a touch screen device. The rotation of the map can also be set through a Shift + click action or by rotating on a touch screen. A north arrow is also provided in the upper right corner which, if clicked, will rotate the map back to true north. The north arrow only appears when the map is not oriented north. The map tools (outlined in red) add functionality for editing and deleting data, and exporting the map to a JPEG file. The routine tool panel (outlined in yellow) houses the functionality to create new airshows, selecting airshows, creating a 
routine, toggling the visibility of maps layers, and enabling the 3D environment. Clicking any of the dropdown menus in this area will reveal the forms required to run each tool.

\subsection{Adding a New Airshow}

Two of the dropdown menus in the routine toolbar on the left open forms for creating a new airshow location and for adding a new "Bomb Burst" location (Figure 6-2).

\begin{tabular}{l|l|}
\hline \multicolumn{1}{|c|}{ Create New Airshow } & \multicolumn{1}{c}{$\begin{array}{c}\text { Create New Bomb Burst } \\
\text { Show Location }\end{array}$} \\
Show Location \\
\hline Nellis AFB & Nellis AFB \\
\hline Date & Latitude \\
\hline 11/14/2015 & Degrees: 36 \\
\hline Latitude & Minutes: 14.269 \\
\hline Degrees: 36 & Longitude \\
\hline Minutes: 14.279 & Degrees: -115 \\
\hline Longitude & Minutes: 1.8911 \\
\hline Degrees: -115 & Submit \\
\hline Minutes: 1.9011 & \\
\hline Submit &
\end{tabular}

Figure 6-2: Input Forms for Creating New Airshow and Bomb Burst Locations

Since the Thunderbirds required that all coordinates be entered in degree decimal minutes, the tools for adding these two points account for the correct input by allowing the user to enter coordinates for degrees and minutes. Degrees should be entered as whole numbers, while minutes should be entered with decimals. Improper coordinate inputs are handled in the JavaScript code by using if statements for the various cases, but inputs should still match this form. Both the airshow point and "Bomb Burst" points are related based on the show location name, which is accepted as the first input for each point.

There can be multiple "Bomb Burst" locations for each show location for analysis 
purposes, but only one may be used at a time for calculating the show routine. A date field is also provided for the show location so that historical show locations can be used.

After filling out the tool parameters for a new point, clicking the submit button will zoom to the location entered in the coordinate fields. By clicking anywhere in the map pane, the new point is then added to the map at that location and saved to the database. Once added to the map, the location of a new point can be edited by clicking the "EDIT" button in the map toolbar. Using this tool allows the user to click and drag a point to a new location. By letting go of the point, the geometry location of the point is updated in the database. Similarly, a point may be deleted from the map and database by clicking the "DELETE" button and then clicking on a point.

\subsection{Creating a New Routine Map}

Creating a map of an airshow routine is the main functionality provided by this application. In the "Select Airshow" dropdown menu on the routine toolbar, a selectable list (Figure 6-3) of all created airshow locations allows the user to select and zoom to an airshow for routine map creation.

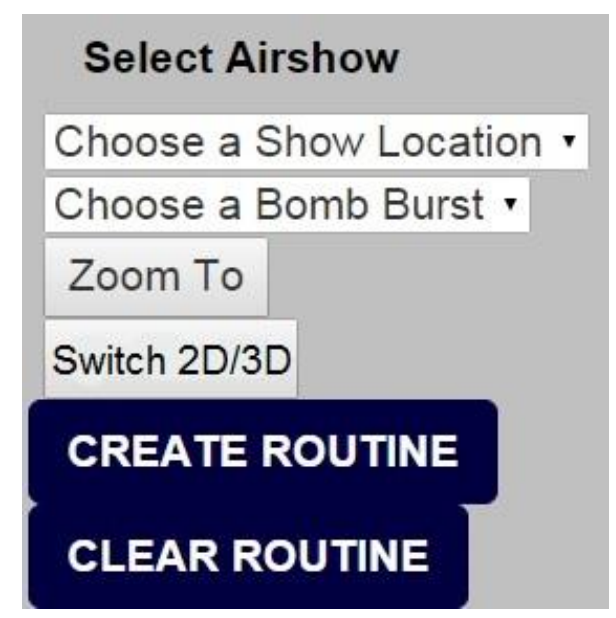

Figure 6-3: Airshow Selection Menu and Create/Clear Routine Buttons 
The related "Bomb Burst" can also be selected from the list below the airshow selection menu. Once the airshow and bomb burst locations have been selected, a new routine map is added by clicking the "Create Routine" button. This adds three layers to the map: maneuver location points, routine overlay, and buildings within the airshow extent (Figure 6-4). The geometries displayed in these three layers are all calculated on the client server and added to temporary layers. Selecting new locations and re-clicking "Create Routine" does not rerun the function. In order to create a new routine map, the "Clear Routine" button must be clicked. This button clears the data from all temporary layers and enables the layers to receive more data when a new routine map is created.

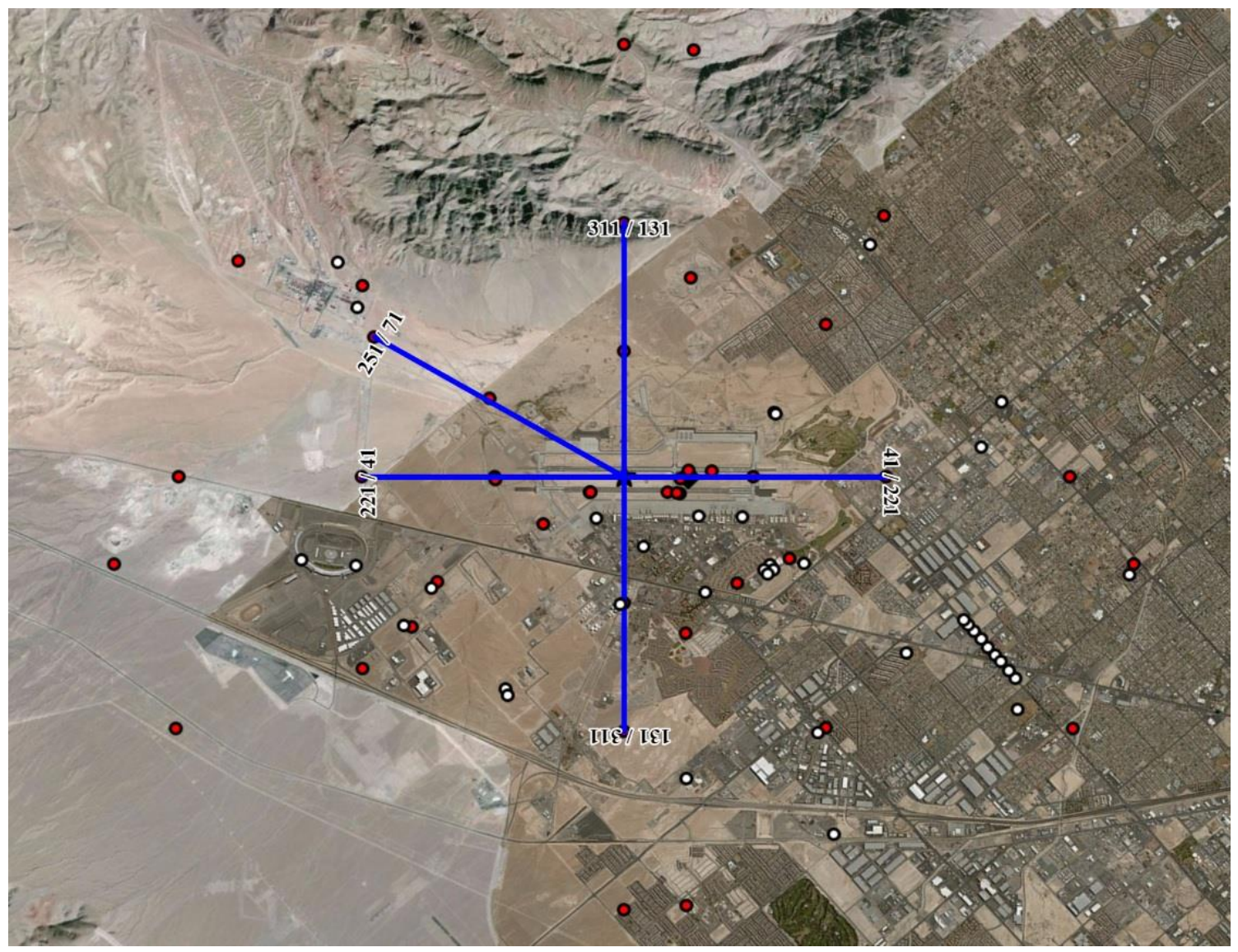

Figure 6-4: Nellis AFB Routine Map. Maneuver Points (red), Routine Overlay (blue), and Buildings (white) 


\subsection{Visualizing a Routine}

The application provides users with options for how they can view the airshows. Show location points and bomb burst locations are always included in the map, but the bomb burst locations are only visible at higher zoom levels. A feature dataset containing the locations of all buildings that pose a potential aerial obstructions is also included in the map when the map loads. All routine layers can be toggled on and off using the check boxes provided in the layers dropdown menu (Figure 6-5). After creating a new routine map, the visibility of all data layers calculated using this function can be turned on and off as well, as to avoid a cluttered or busy map.

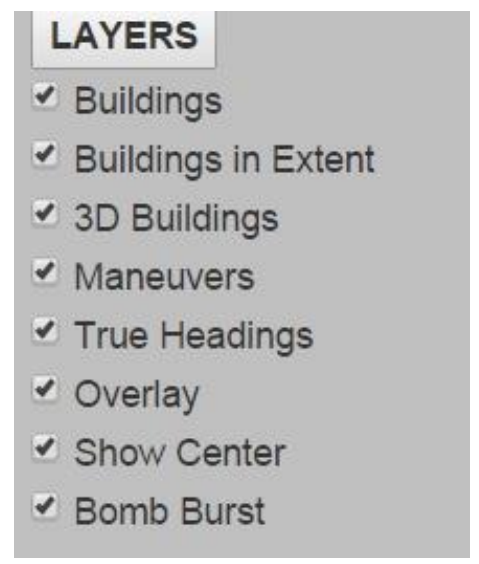

\section{Figure 6-5: Application Layers Menu}

The application also integrates a 3D environment for data visualization. Clicking the "Enable 3D" button (in Figure 6-3) turns on the 3D scene (Figure 6-6), automatically loading the terrain model and applying a z-value for all geometries. This z-value gives height information for points, lines, and polygons. Extruded buildings and routine point buffers are automatically added to the map. The view angle for the scene when loaded looks straight down at the airshow routine. The view angle can be changed with a Shift + click command on the map; dragging down on the map increases the view angle, giving 
shape and height to the terrain. This effect can be replicated on a touch screen device by simultaneously moving two fingers in the same direction. Other navigation functionality remains in the 3D environment except double clicking to zoom in. Clicking the "Switch $2 \mathrm{D} / 3 \mathrm{D}$ " button returns the scene to a $2 \mathrm{D}$ view for all data.

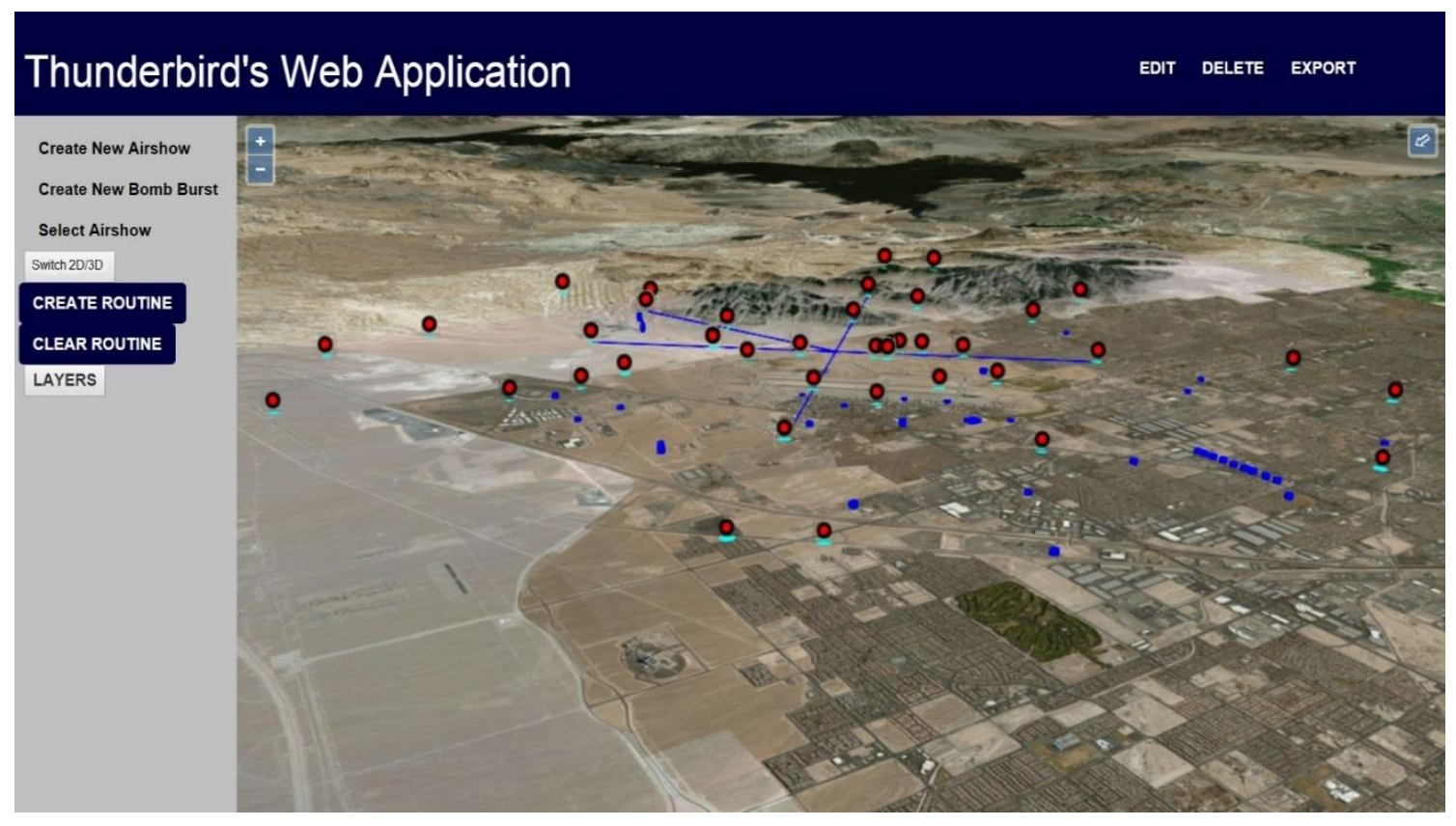

Figure 6-6: Airshow Routine Displayed in 3D Environment

\subsection{Sample Locations}

In order to compare the application results to the maps produced by the Thunderbirds, two sample sites were selected: Nellis AFB and Creech AFB. The comparison of the produced maps can be seen in Figure 6-7 and Figure 6-8. 


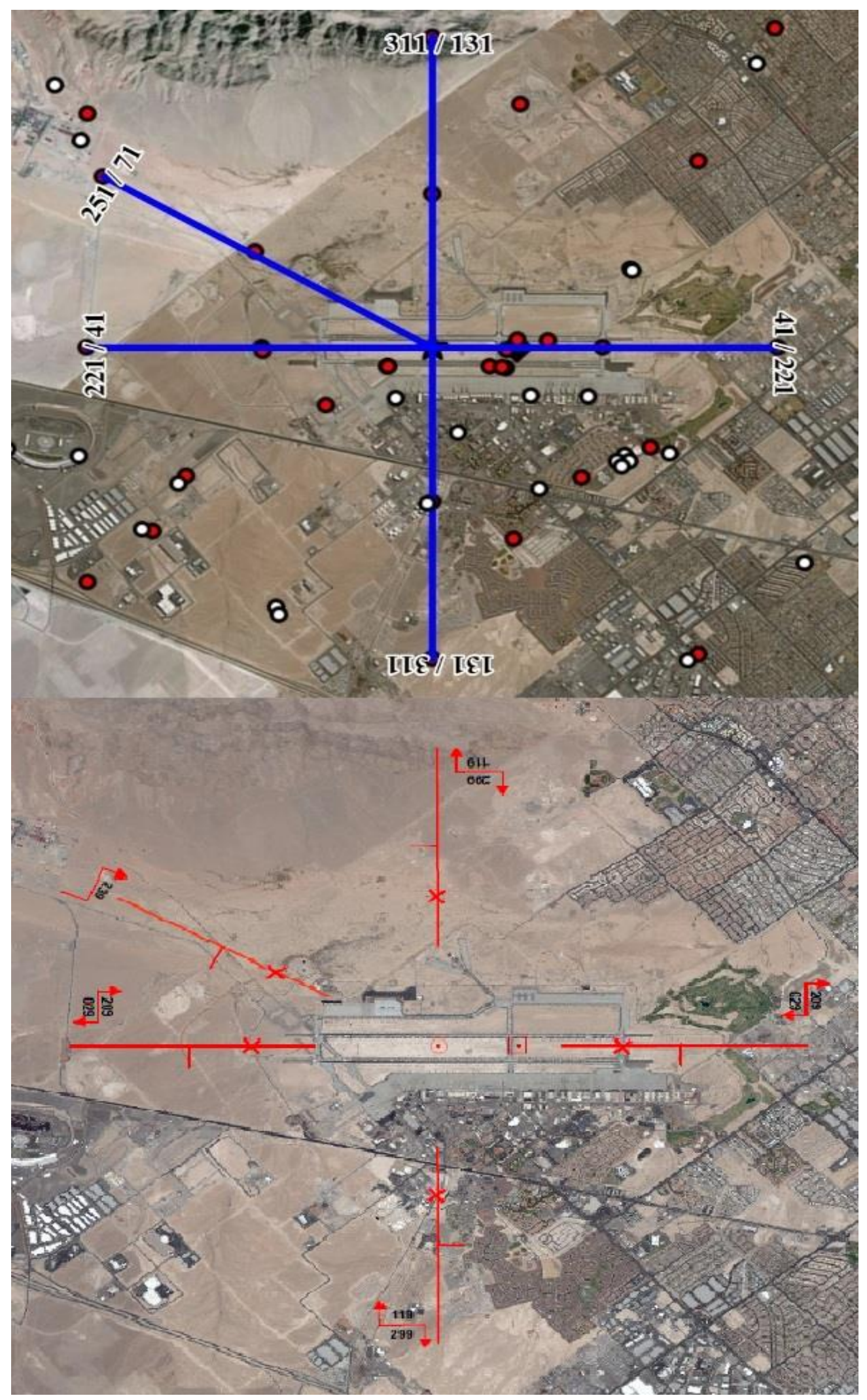

Figure 6-7: Comparison of Application (top) and Sample (bottom) at Nellis AFB 


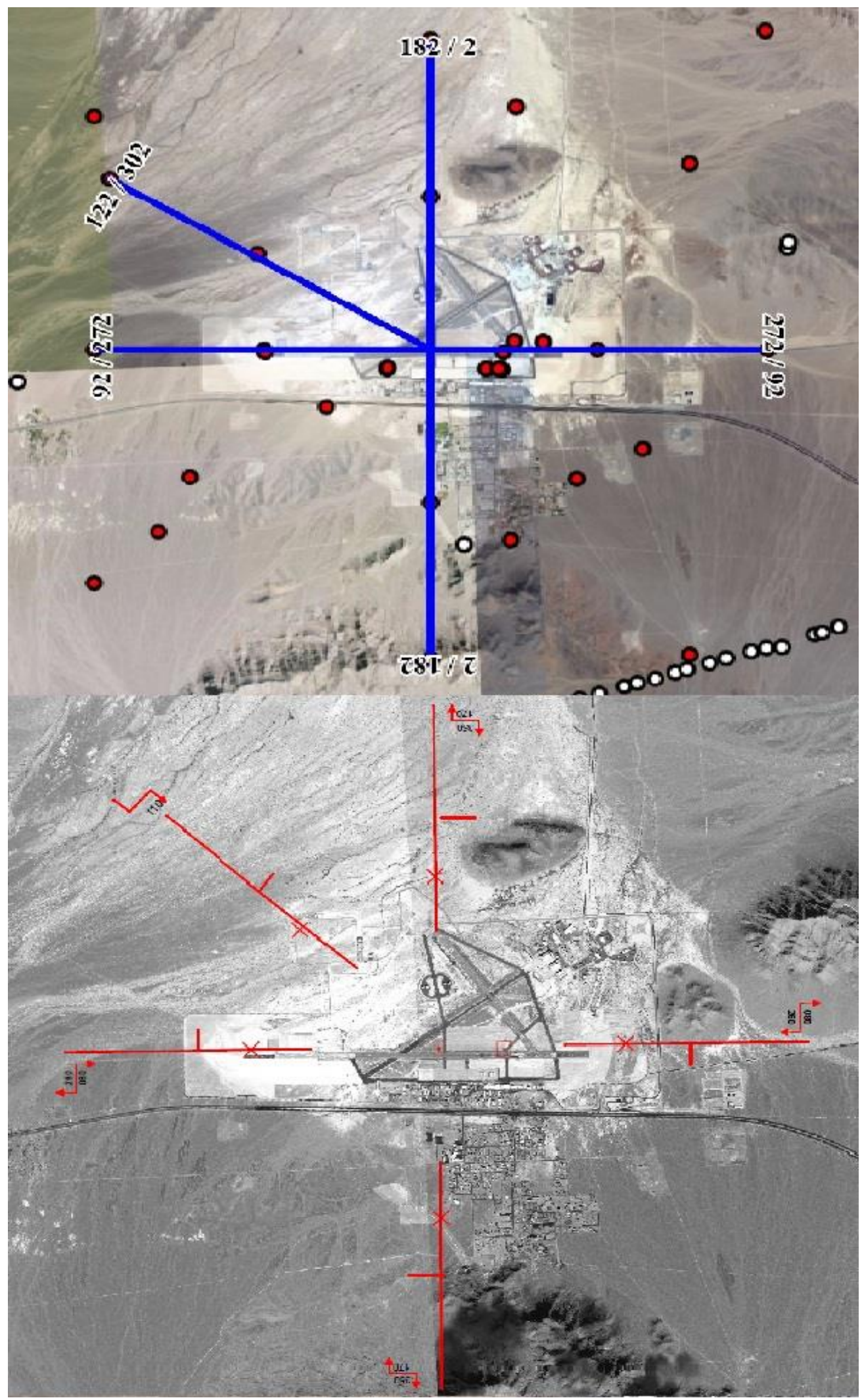

Figure 6-8: Comparison of Application (top) and Sample (bottom) at Creech AFB 
The results from the application map when compared to the sample maps shows the accuracy of the application. In addition to creating the same routine overlay, the application maps also include maneuver point locations and building locations. The inbound and outbound angles are slightly different, however. The sample maps used magnetic heading, while the application uses true heading. Magnetic heading (based on the magnetic north) can be found from the true heading (based on the true north) by adding the magnetic declination. The magnetic declination is different on all parts of the globe and most pilots use their flight computers to calculate this angle. The Thunderbirds can use the true heading in the application maps to find the magnetic heading.

When comparing the two maps from Creech AF, there appears to be a difference in angles on the left side of the map. The original map provided by the Thunderbirds appears to be at more of a 45 degree angle, while the application maps shows a 30 degree angle. However, if you take a closer look at the stated headings for each line in the original map, the separation is actually a 30/60 degree change. In this case, the two maps are likely in different projections.

Even though the Thunderbirds do not traditionally perform at the City of Redlands Airport, a map was created in the application to show how the Thunderbirds can create airshow maps anywhere around the United States. Figure 6-9 shows the routine map for the City of Redlands Airport that was created in the application. The terrain to the north of the airfield contains mountains and hills, so the Thunderbirds would most likely change the Show Center and/or Bomb Burst locations so that their routine is safe. 


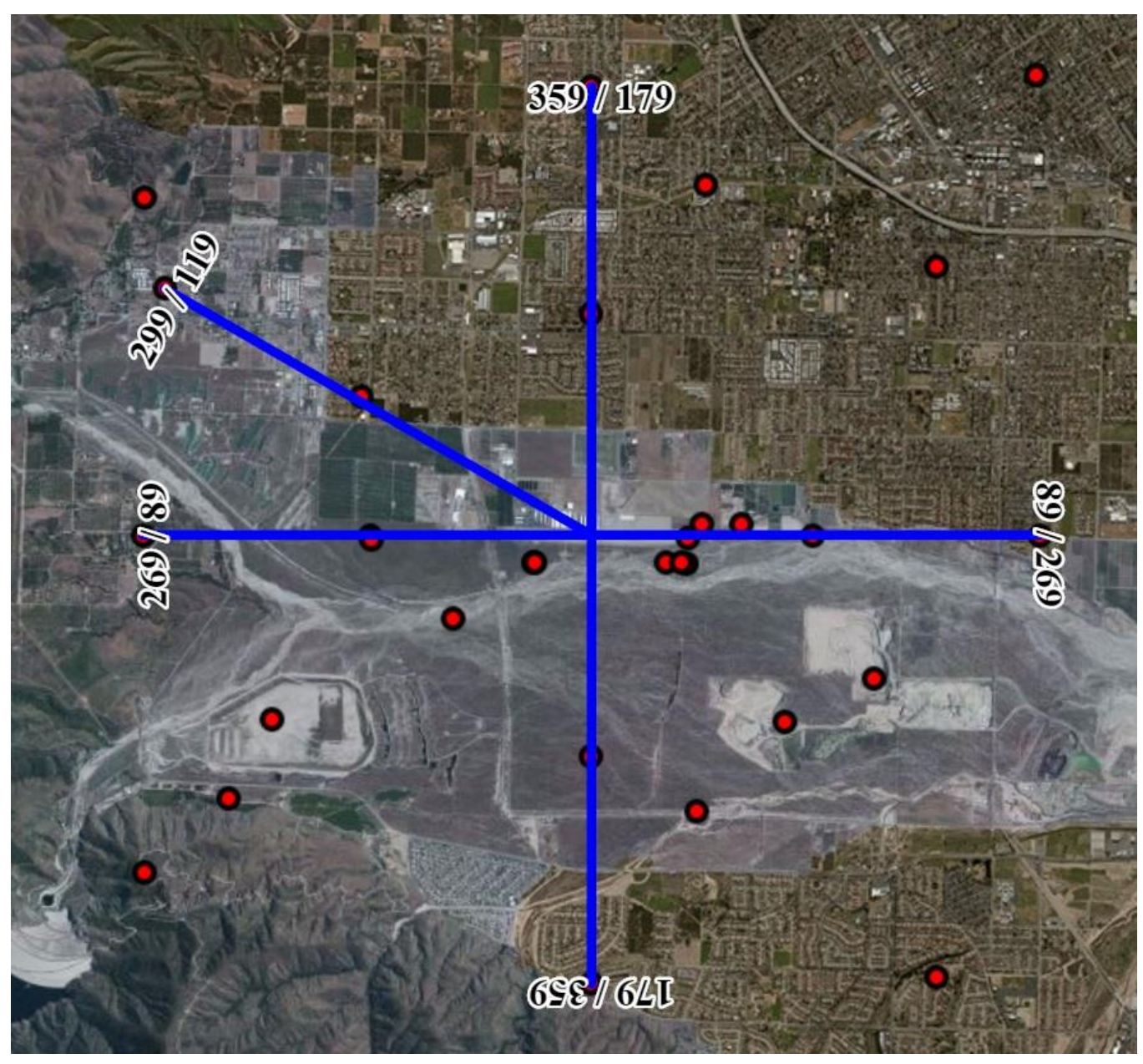

Figure 6-9: Routine Map for Redlands Airport

\subsection{Summary}

The Thunderbirds Routine Mapping Application provides a lot of functionality for the

Thunderbird pilots. After adding new airshows to the database, a routine map of the airshow can be created and displayed. Layers of the maneuver points, overlays, and buildings are visualized in the routine map. Finally, the map can be loaded into a 3D visualization allowing the pilots to navigate through their routine, searching for potential safety hazards. 



\section{Chapter 7 - Conclusions and Future Work}

\subsection{Summary}

The United States Air Force Thunderbirds perform in airshows all around the world, drawing large crowds as they travel. A lot of work goes into the planning of each airshow, since the Thunderbirds need to adhere to regulations in order to protect the pilots and those enjoying the show. In an effort to speed up this process while at least maintaining the same amount of information needed, the Thunderbirds reached out for a mobile solution that allowed the pilots to create, edit, and view their routine maps. This project accomplished those goals, and provided additional analysis functionality by creating a mobile web application that dynamically creates the airshow routine map based on user input coordinates for the show location. The following functionalities were implemented:

- Edit and delete routine point locations through database transactions

- Display headings of flight angles

- Export map to a JPEG file

- Turn layers on and off for optimal analysis

- Safety analysis by adding the location of all building obstructions and a 50 meter buffers around maneuver points

- 3D environment with height, extrusion, and terrain visualization 
This application was created with the use of the open source software provided with the OpenGeo suite. Three datasets were created and hosted on the web with the use of PostgreSQL and GeoServer. The application was scripted with JavaScript, HTML, and CSS with the libraries provided by the OpenLayers 3 and OpenLayers Cesium APIs. A Python tool was scripted to calculate the distances and azimuths of each routine point relative to the show center location of a sample dataset, which was then used to dynamically calculate and plot routine point locations for each new airshow. With just a few minutes of work, the application allows the Thunderbirds to finish a process that used to take them three hours.

\subsection{Future Works}

Even though this project added some analytical functionality to the planning process of the Thunderbirds, there are a few ways that this project could be expanded in the future, most of which has to do with 3D visualization. In that environment, the 3D view of building obstruction data was simplified to point locations based on the limitations of the data. Expanding this by applying classifications for building type and applying realistic building texture to the extrusions would allow for better visualization of the routine scene.

A tool could also be incorporated into the $3 \mathrm{D}$ environment for visualizing the sun based on the time of day, allowing the pilots to look for any potential sun glares that they

might have to deal with. Another expanded functionality that could be built within the 3D viewer is a fly through of the routine. Programmatically, the scene camera angle, position, and view extent can be changed in a function with a predefined route, allowing the viewer to watch a video of the routine in a "rollercoaster view". This could be done 
from a $1^{\text {st }}$ or $3^{\text {rd }}$ person perspective. A Thunderbird F-16 jet could also be modeled in the 3D scene and be used as part of the fly through. 



\section{Works Cited}

ArcNews Spring 2011. (n.d.). Retrieved November 24, 2015, from

http://www.esri.com/news/arcnews/spring 11articles/open-source-technology-andesri.html

Brail, R., \& Klosterman, R. (Eds.). (2001). Planning support systems: Integrating geographic information systems, models, and visualization tools. ESRI Press.

Education Center - Boundless. (2015). Retrieved August 20, 2015, from

http://workshops.boundlessgeo.com/suiteintro/

Esri. (2015). Design and create Mobile maps. Retrieved from ArcGIS Desktop Help: http://webhelp.esri.com/arcgisdesktop/9.3/index.cfm?TopicName=Design_and_creat e_Mobile_maps

Fu, P., \& Sun, J. (2011). Web GIS: Principles and applications. ESRI Press.

Fu, P. (2015). Getting to know web GIS. ESRI Press.

Knox, D. (2004). 3D Terrain Visualisation and Virtual Fly-Through for Tourism Conservation Using Geographic Information Systems (GIS). Retrieved from https://eprints.usq.edu.au/41/1/DonnaKNOX-2004.pdf

Mojave Desert Ecosystem Program. (2015). About. Retrieved from Mojave Desert Ecosystem Program Website: https://www.mojavedata.gov/about.html

Nishii, Chisa. (2007). Web-GIS Services for Sustainable Ecotourism: Protected Areas in Northern Honduras (Master's thesis, University of Redlands). Retrieved from http://inspire.redlands.edu/gis_gradproj/98

OSGeo FAQ. (2015). Retrieved November 24, 2015, from http://www.osgeo.org/content/faq/foundation_faq.html 
Ramroop, S. (2006). 3-D Development Case Study at Troy University. Retrieved from http://proceedings.esri.com/library/userconf/proc06/papers/papers/pap_1139.pdf

Robinson, D. (2009). GIS for Airports - Electronic Terrain and Obstacle Data Model (Master's thesis, University of Redlands).

Shinde, S. (2013). Vector-based Mobility Modeling (Master's thesis, University of Redlands).

Sickle, J. (2004). Basic GIS coordinates. CRC-Press.

Thunderbirds History. (2015). Retrieved November 10, 2015, from http://aerobaticteams.net/thunderbirds-history.html

Torres-Ruiz, G. (2009). Web-based GIS Application for Dissemination of Geodetic Data of Military Airfields (Master's thesis, University of Redlands).

United States Air Force. (2015). Retrieved December 4, 2015, from http://afthunderbirds.com/site/history/

United States Air Force. (2015). Retrieved December 4, 2015, from http://afthunderbirds.com/site/our-team 


\section{Appendix A. Nellis AFB Sample Routine Map}

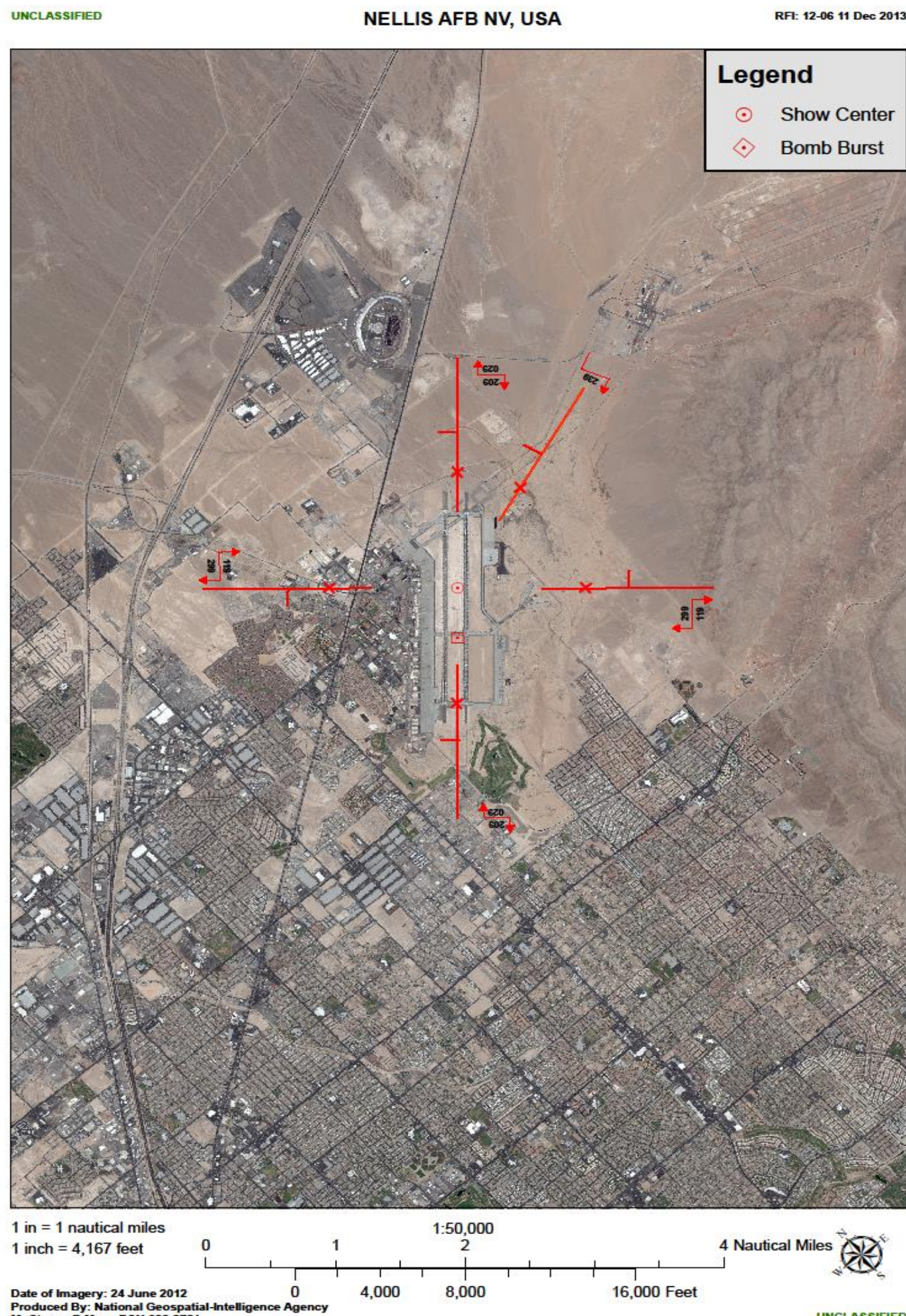




\section{Appendix B. Creech AFB Sample Routine Map}

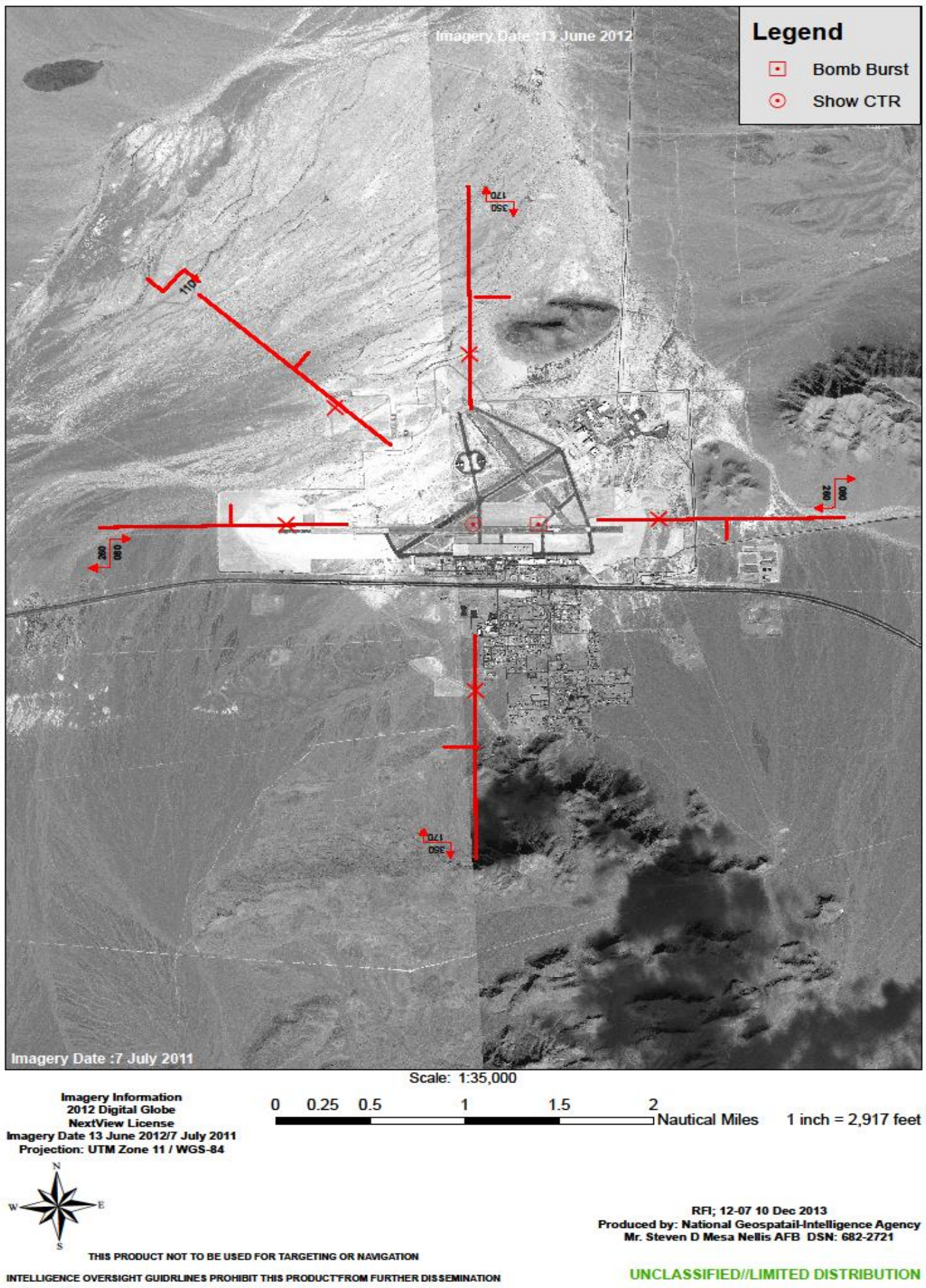




\title{
Appendix C. Distance and Angle Python Script
}

\author{
DistAngle.py \\ \# Created by: Alex Strobl \\ \# Distance and Azimuth Angle \\ \# Created on: 2015-10-13 \\ \# Description: Takes input coordinates and measures distance and angle from \\ \# show center point for a sample Thunderbirds Routine Map \\ \# \\ \#import module \\ import math \\ \#set center point coordinates \\ centerlat $=$ math. radians $(36.238)$ \\ centerlong $=$ math $\cdot$ radians $(-115.031667)$ \\ \#input point coordinates \\ pointlat = float (raw_input ("Enter latitude of the point in decimal degrees: ")) \\ pointlong = float(raw_input("\nEnter longitude of the point in decimal degrees: ")) \\ radlat $=$ math . radians (pointlat) \\ radlong $=$ math . radians (pointlong) \\ \#calculate distance \\ dlong = radlong - centerlong \\ dlat = radlat - centerlat \\ $\mathbf{R}=6373$ \#Earth's average radius in kilometers \\ $a=$ math.sin $(\mathrm{dlat} / 2) * * 2+$ math. $\cos ($ centerlat $) *$ math. $\cos ($ radlat $) *($ math.sin $(\mathrm{dlong} / 2)) * * 2$ \\ $c=2 *$ math. atan2(math.sqrt(a), math.sqrt (1-a)) \\ dist $=R * C$ \\ $\mathrm{ft}=$ dist $/ 0.0003048$ \\ print "The distance in $\mathrm{km}$ is " + str(dist) + "\nor in feet is " $+\mathrm{str}(\mathrm{ft})$ \\ \#calculate azimuth angle \\ theta $=$ math. $\operatorname{atan} 2$ (math.sin (radlong-centerlong) ${ }^{*}$ math.cos (radlat), math. cos (centerlat) \\ *math.sin (radlat)-math.sin(centerlat)*math. cos (radlat)*math. cos (radlong-centerlong)) \\ deg $=$ (math. degrees $($ theta $)+360) \% 360$ \\ print str(deg)
}




\section{Appendix D. OpenLayers 3 JavaScript Functions}

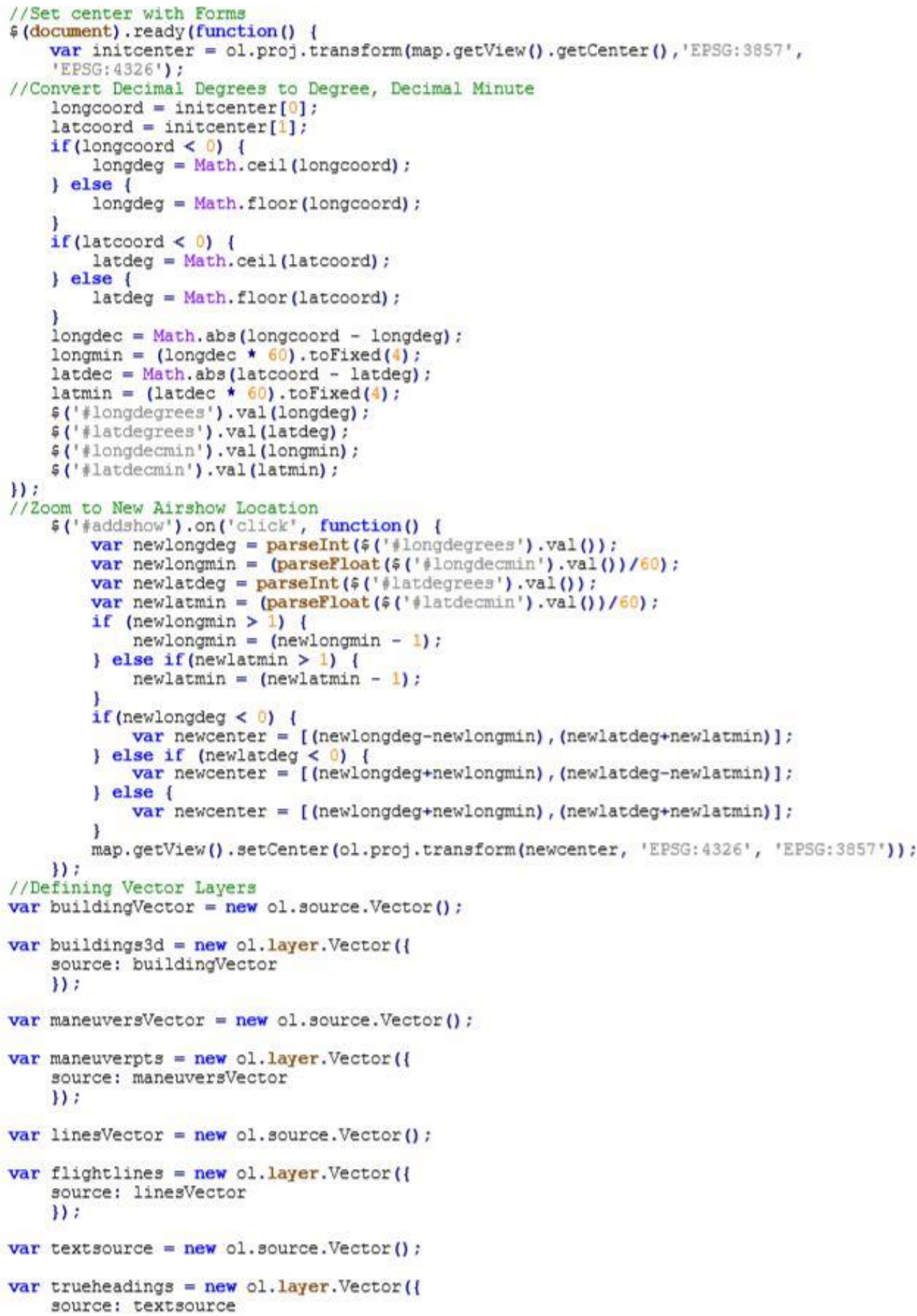




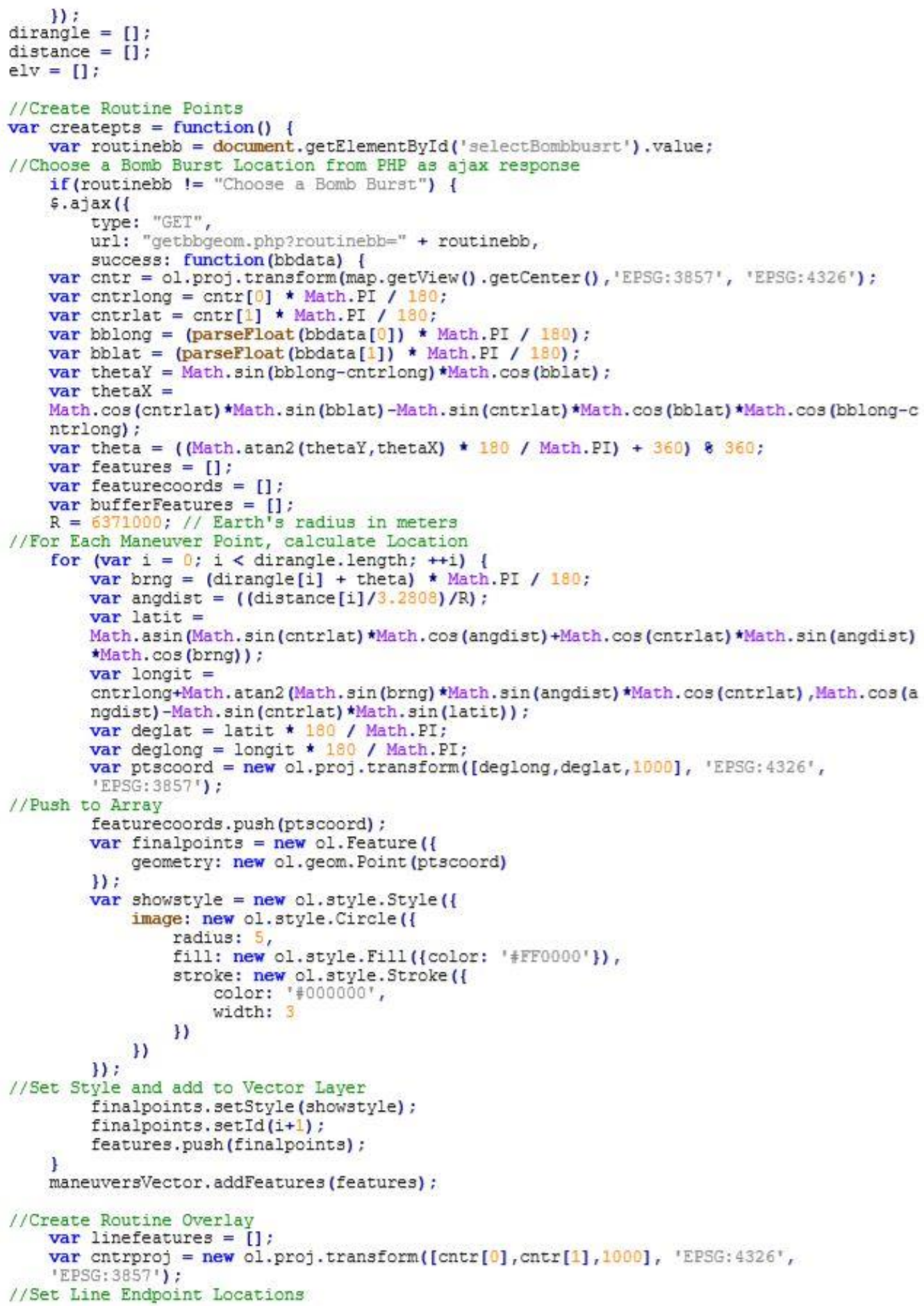




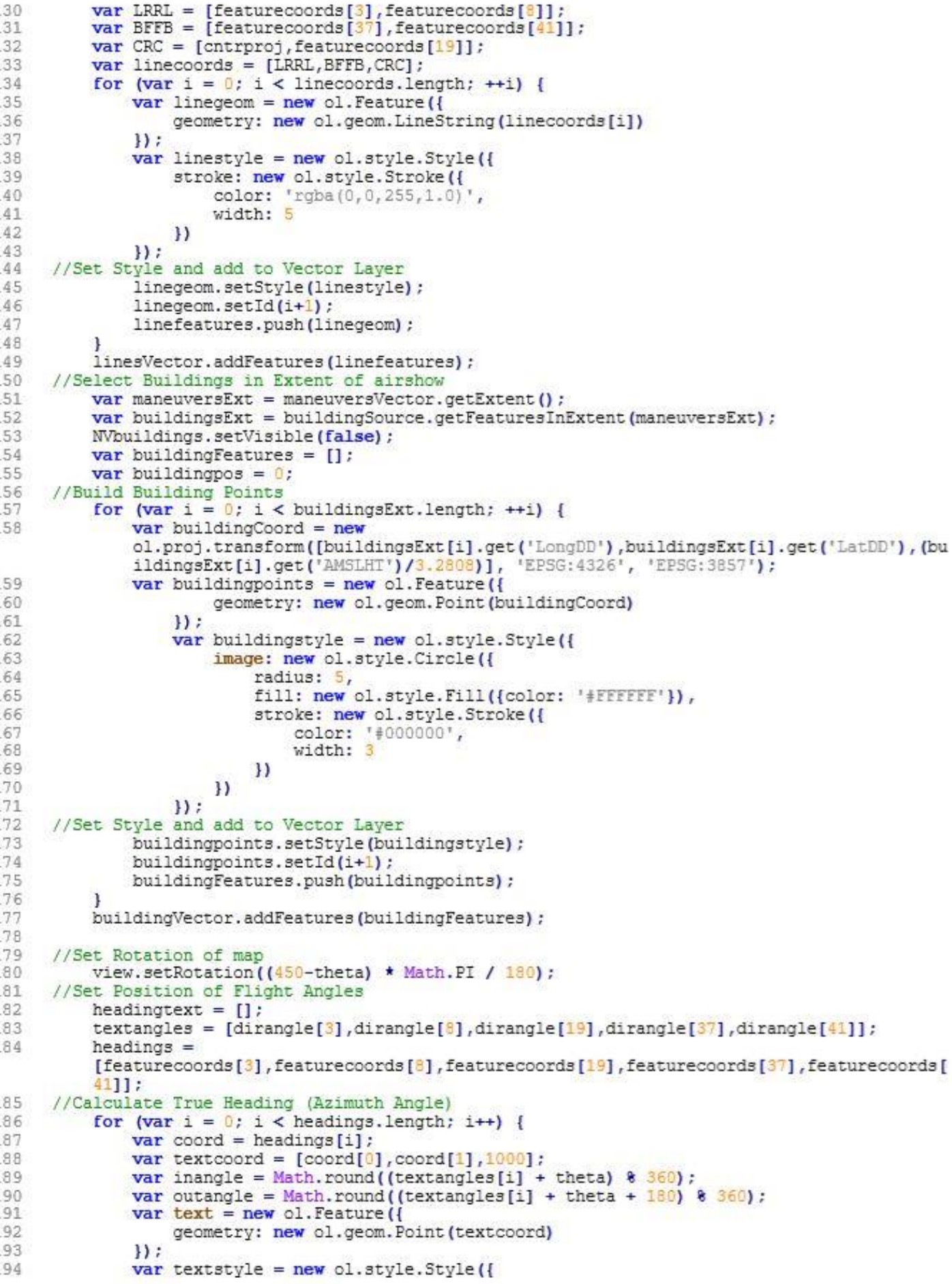




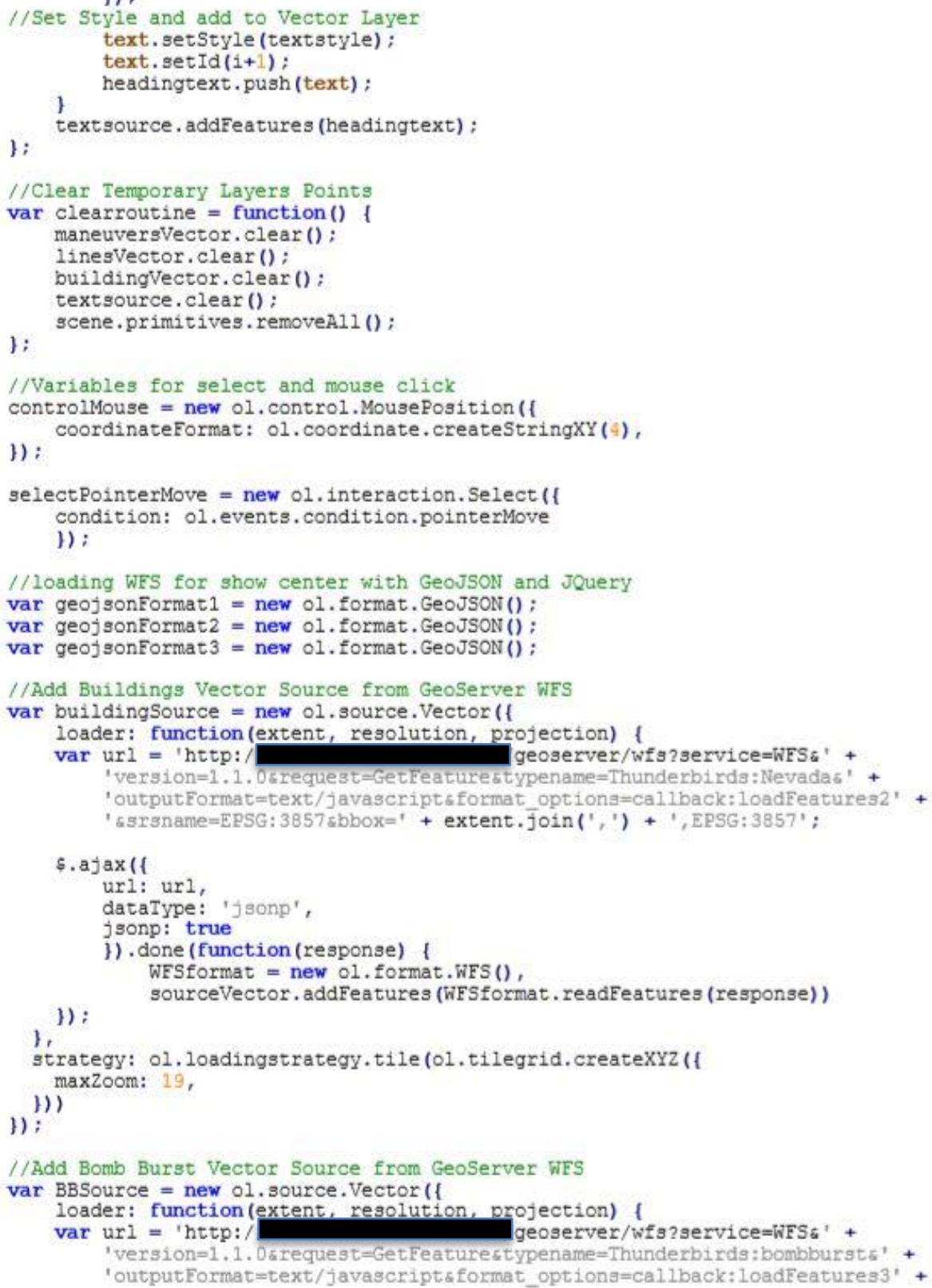
) ;

tyle and add to Vector Layer text.setstyle (textstyle); 


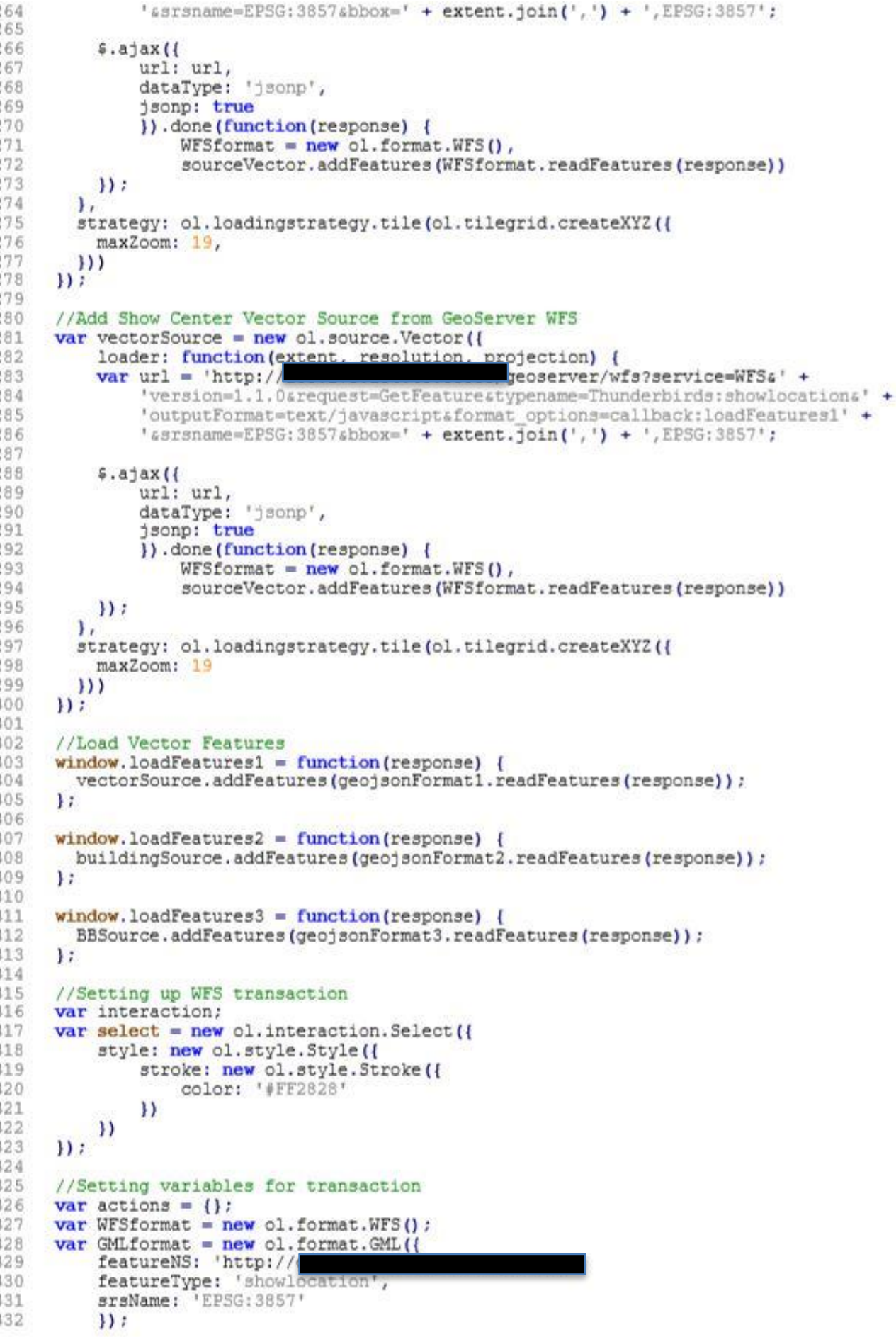




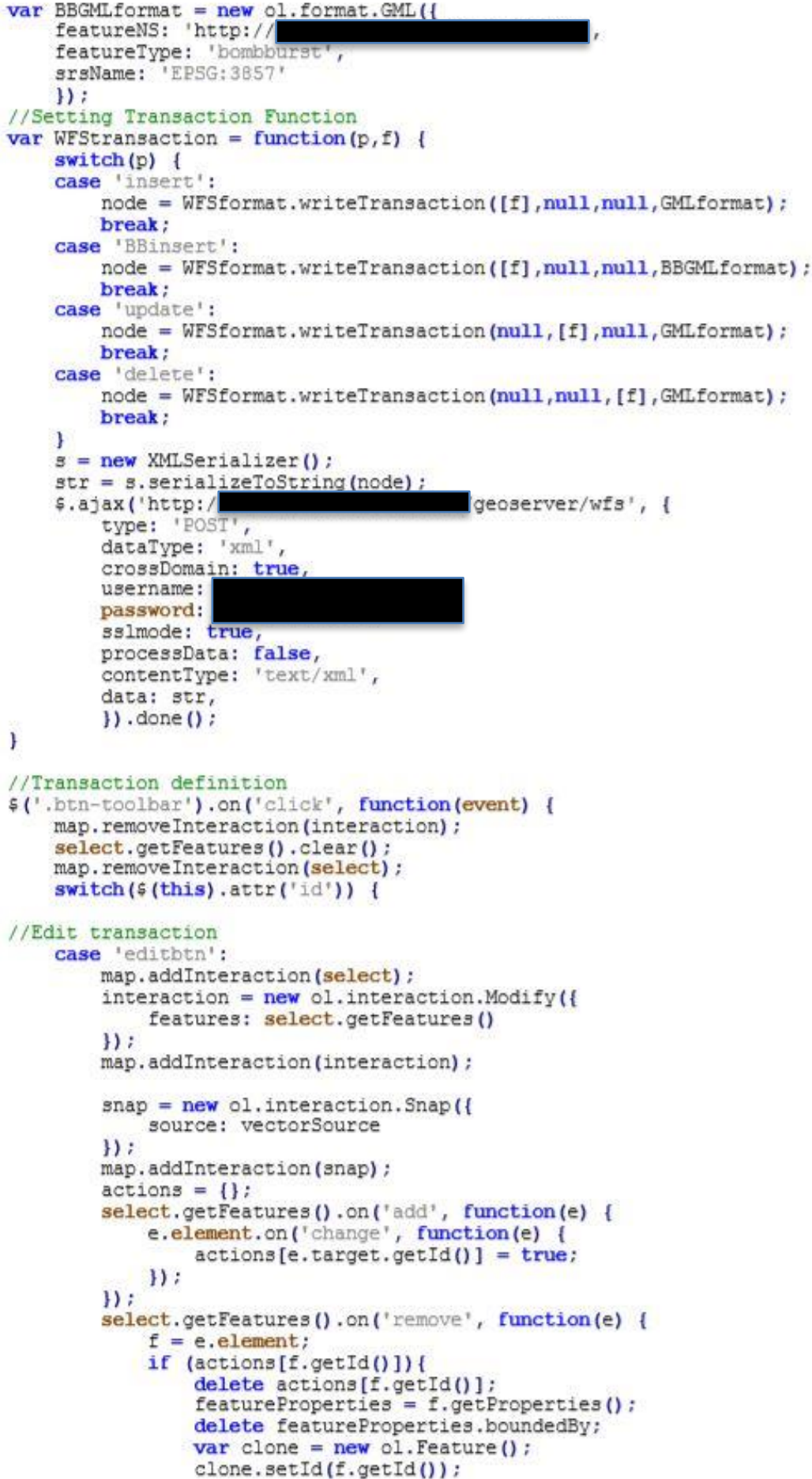




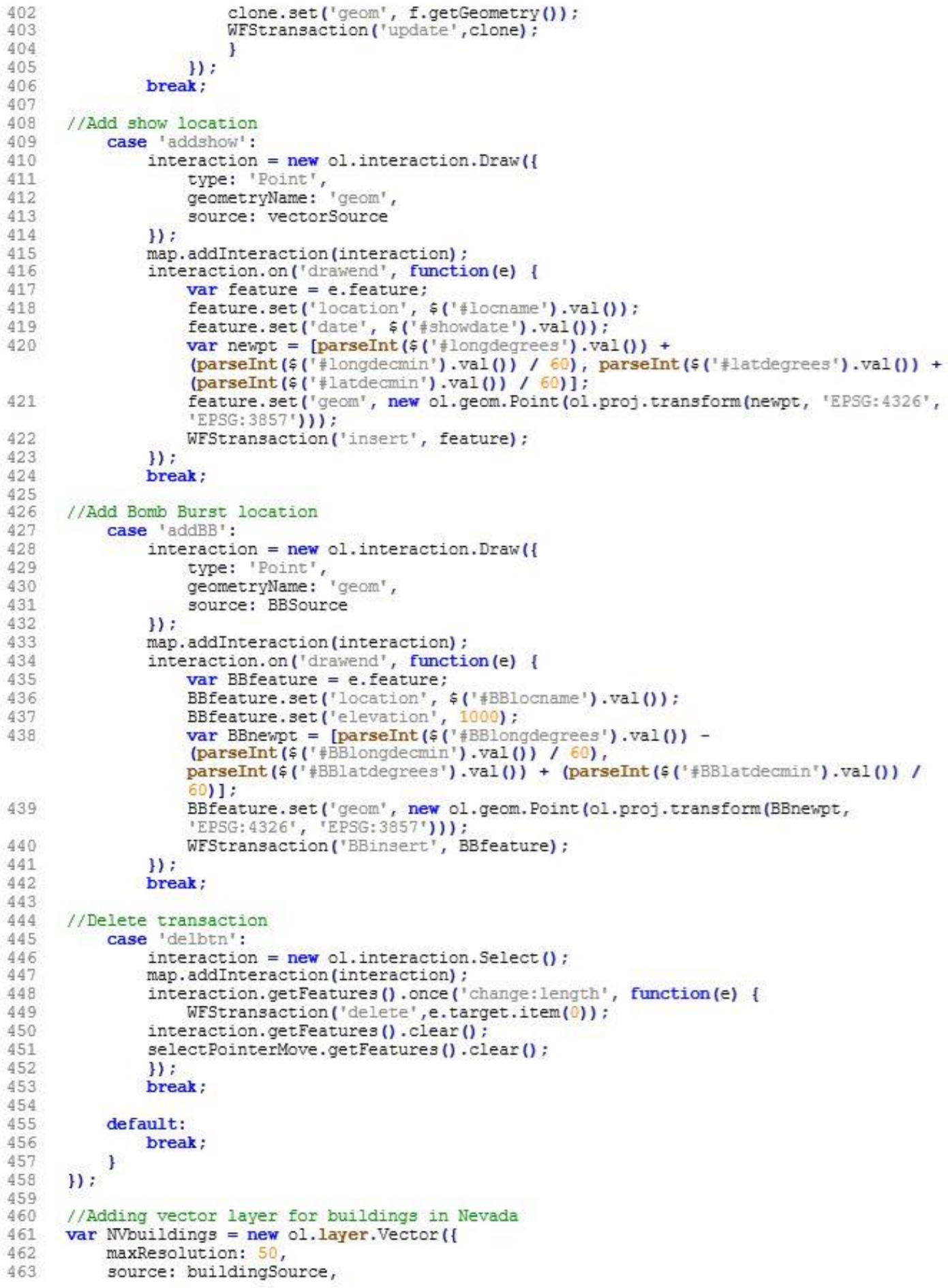




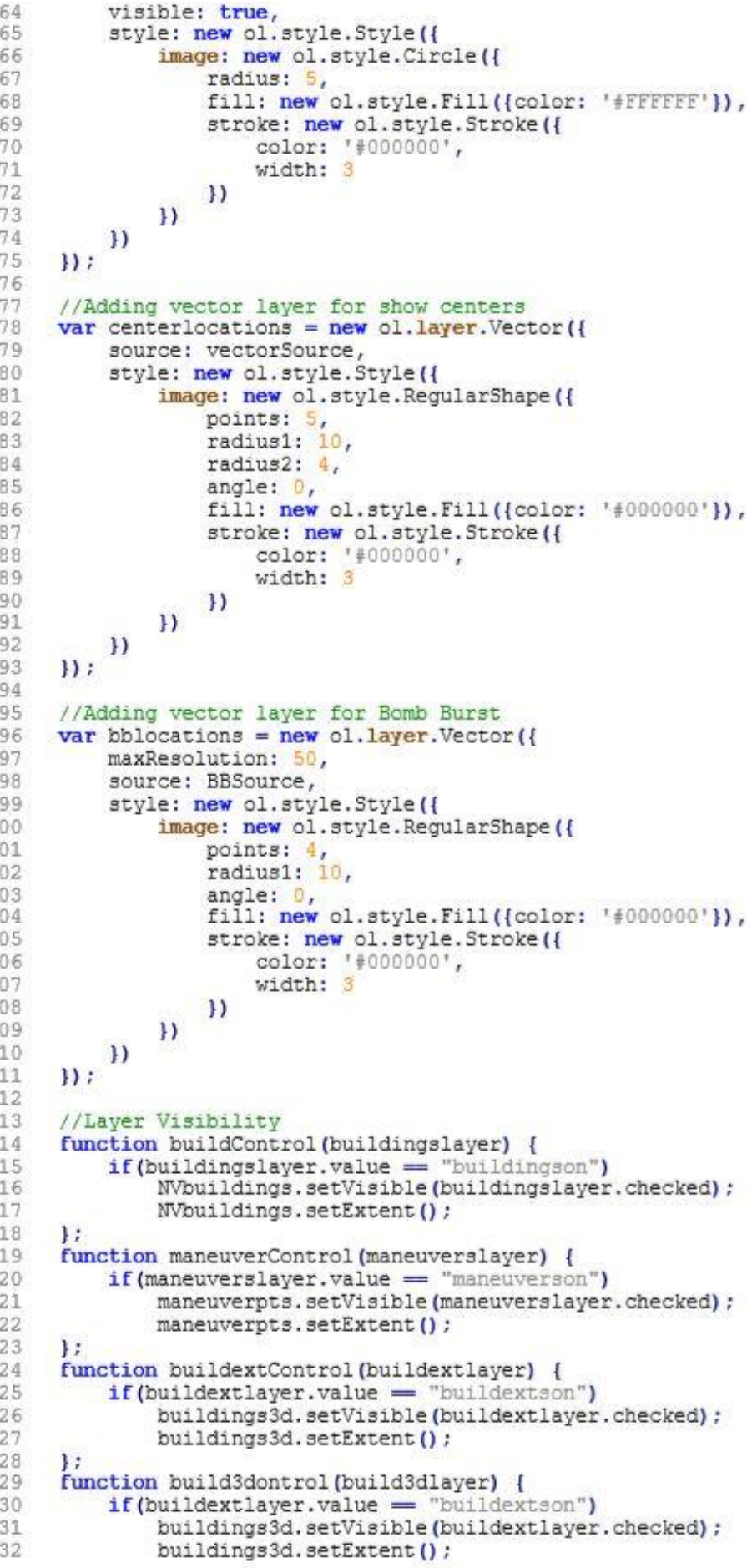




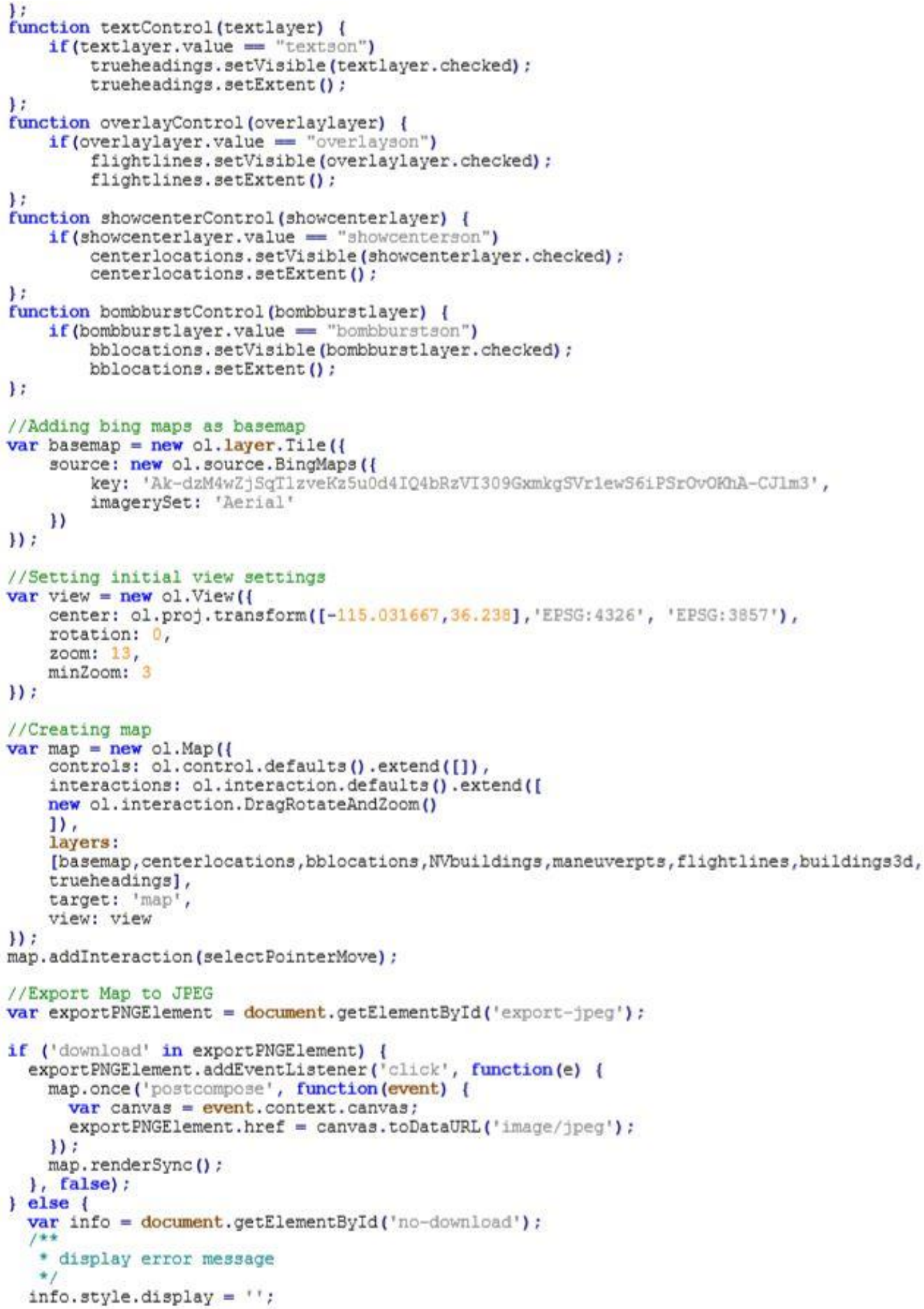


6001 


\section{Appendix E. Cesium JavaScript Functions}

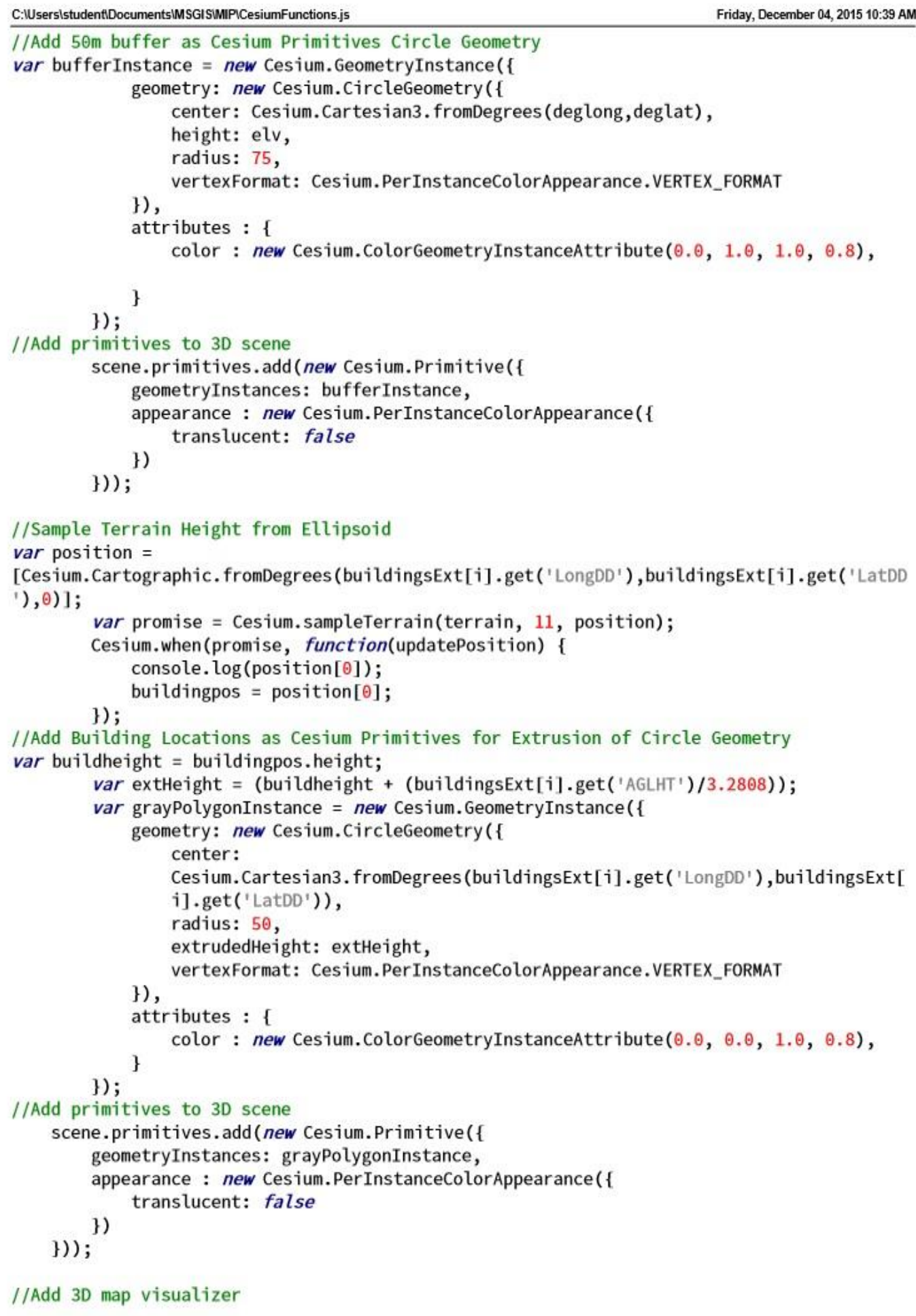


C:IUsersistudentiDocumentsIMSGISMIPICesiumFunctions.js

var ol3d = new olcs. OLCesium (\{map: map\});

var scene $=$ ol3d.getCesiumScene () ;

var globe = scene. globe;

globe. depthTestAgainstTerrain = true;

var terrain $=$ new Cesium. CesiumTerrainProvider $(\{$

\}); url: '//assets.agi.com/stk-terrain/v1/tilesets/world/tiles'

scene. terrainProvider = terrain;

ol3d.setEnabled ( false); 


\section{Appendix F. User Guide}

\section{Airshow Routine Mapping Application}

\section{USER MANUAL}
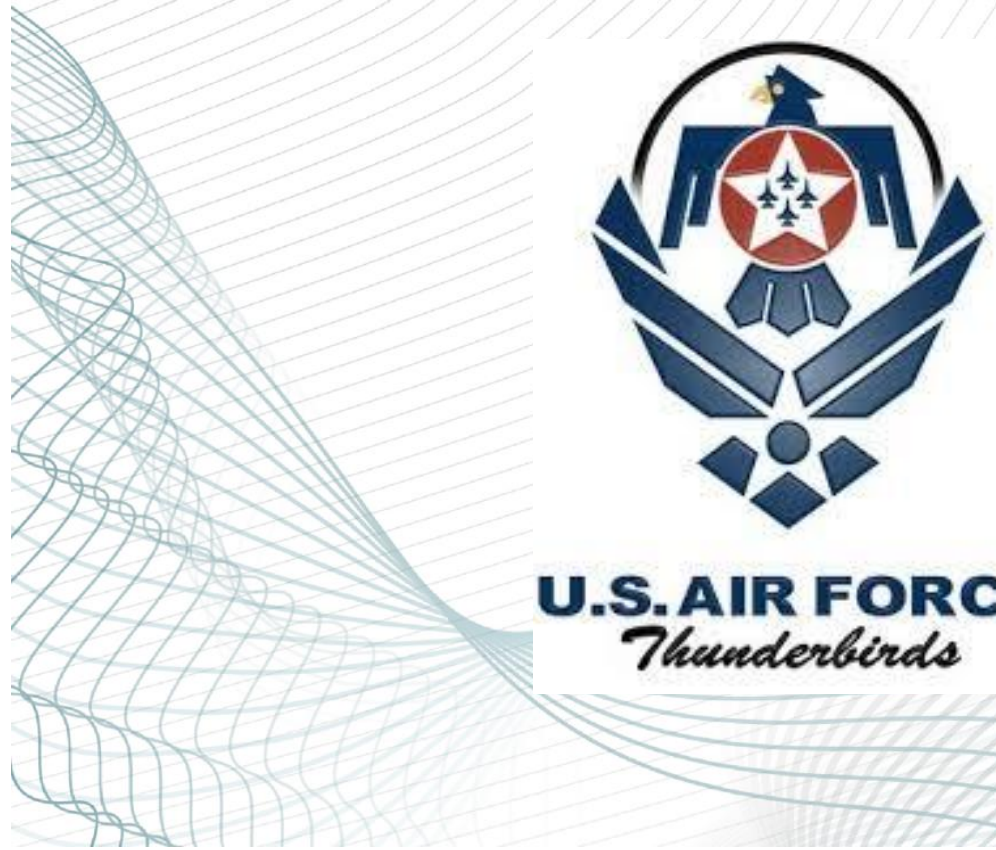

U.S.AIR FORCE Thusederbirds 


\section{Table of Contents}

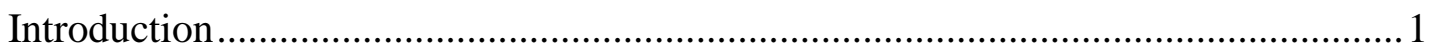

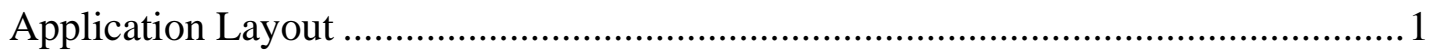

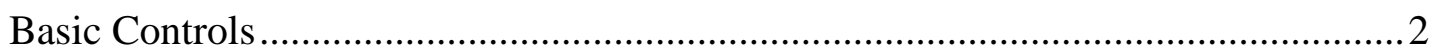

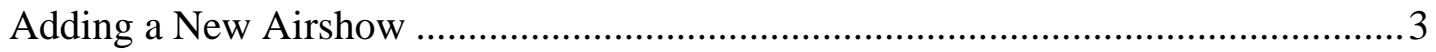

Creating a New Routine Map .................................................................. 4

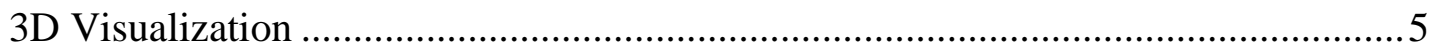




\section{Introduction}

Welcome to the U.S. Airforce Thunderbirds Routine Mapping Application. This User Manual provides instructions for quickly mapping out the Thunderbirds airshow routine at any location around the world and explores the various functionalities of the application.

The Routine Mapping Application can be accessed through any web browser or mobile device. A Common Access Card (CAC) can provide fast access through a desktop computer, but you can also log in using provided username/password combinations. A username and password are required for accessing the application though a mobile device.

The application will automatically plot the location of your airshow maneuver locations based on the input of show location and Bomb Burst. Users can explore the location to check for safety hazards, including buildings and terrain, in 2D and 3D views.

\section{Application Layout}

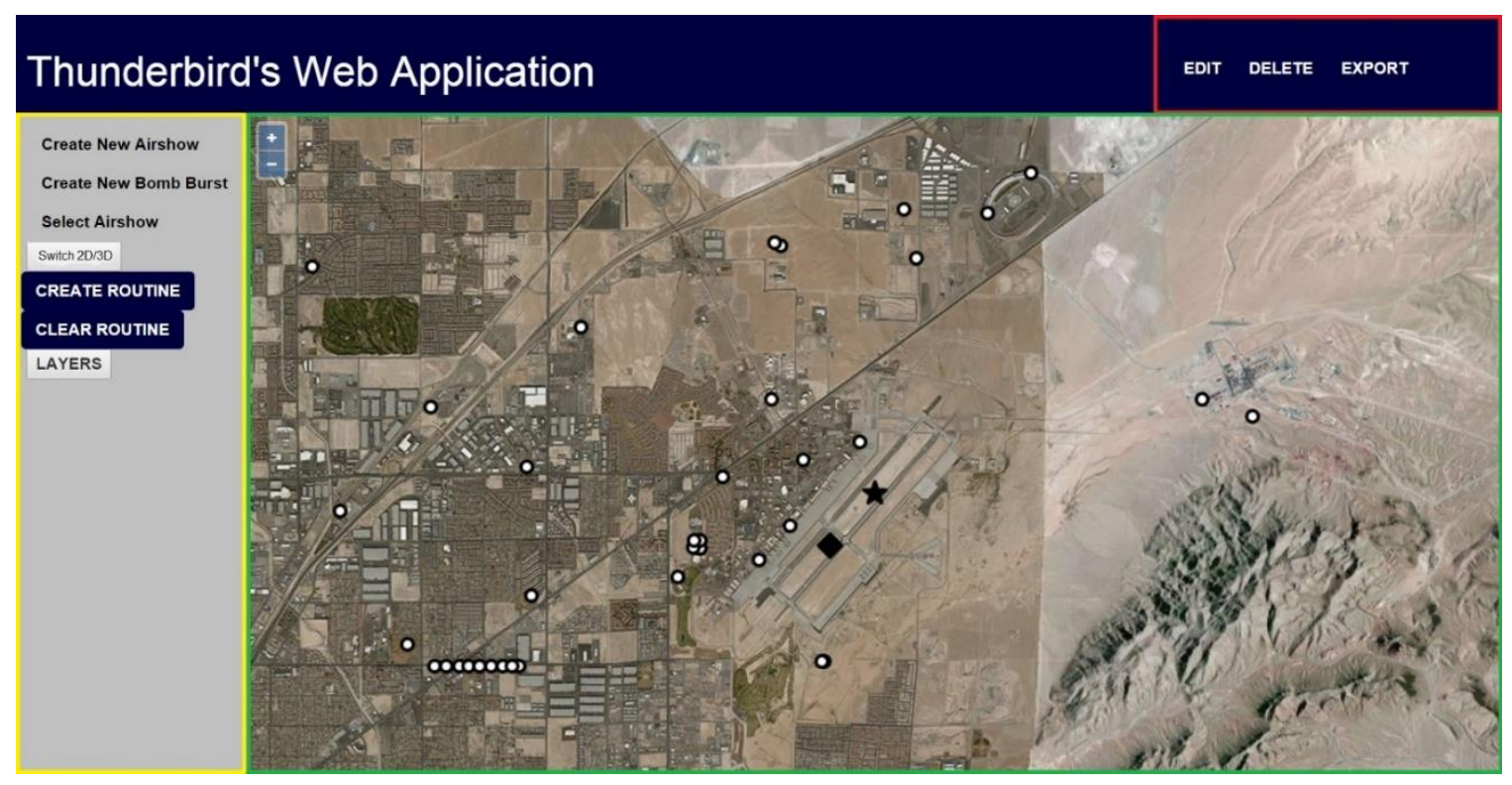


This section will cover the layout of the application, which is broken down into three sections:

1) Map Viewer (Green Box): This section displays all data layers for the application, including the $3 \mathrm{D}$ viewer, and allows the user to explore the airshow routine and surrounding area.

2) Map Tools (Red Box): Edit and delete data in the Map Viewer. The Map Viewer can also be exported to a JPEG.

3) Routine Tools (Yellow Box): stores the functionality to create new airshows, changing the selected airshow, creating a routine, toggling layer visibility, and view the $3 \mathrm{D}$ environment.

\section{Basic Controls}

- Zoom In/Out: click the "+,,“" buttons on the left side of the map viewer or double clicking in the map viewer

- Pan Map: click and drag image

- $\quad$ Rotate Map Viewer Orientation: press and hold SHIFT. Then click and drag the map viewer to rotate the imagery

- For Mobile Users: your fingers can be used to zoom in/out, pan map, and rotate the map viewer

- North Arrow: located in the upper right side of the map viewer, the North Arrow will always point North. If clicked, the orientation of the map returns to default

- Edit a Data Point: click EDIT in the map tools pane. Then click the desired data point to select. Once selected, the point can be moved to a new location 
- Delete a Data Point: click DELETE in the map tools pane. Then click the desired data point. Click again to delete.

- Export Map: click EXPORT in the map tools pane. A snapshot of the map viewer will save as a JPEG to your Downloads folder

- Switch Map Viewer to 3D: click the SWITCH 2D/3D button in the routine tool panel. The map viewer will toggle between the two visualizations

- Layers: clicking LAYERS in the routine tool panel will display all data layers loaded in the map viewer. Uncheck a box to remove that data layer from the map viewer

\section{Adding a New Airshow}

Follow the steps below for adding a new airshow to the application database:

1) Click Create New Airshow

2) Enter the location name and show date in the appropriate fields

3) Enter the location of the show center point in degree decimal minutes

4) Click Submit to save the airshow location. The map viewer will zoom to this location

5) Click Create New Bomb Burst

6) Enter the airshow location name (note: multiple Bomb Burst can be created per airshow, but only one can be used to generate an airshow map)

7) Enter the location of the Bomb Burst in degree decimal minutes

8) Click Submit to save the Bomb Burst location 


\begin{tabular}{l|l|}
\multicolumn{1}{|c|}{ Create New Airshow } & \multicolumn{1}{c}{$\begin{array}{c}\text { Create New Bomb Burst } \\
\text { Show Location }\end{array}$} \\
Show Location
\end{tabular}

\section{Creating a New Routine Map}

Follow the steps below for creating a new airshow routine map:

1) Click Select Airshow in the routine tool panel

2) Select a Show Location and appropriate Bomb Burst location in the dropdown menus

3) Click Create Routine. This will add three layers to the map viewer: maneuver location points, routine overlay, and buildings within the airshow extent

4) Clicking Clear Routine will remove airshow routine layers and reset the application

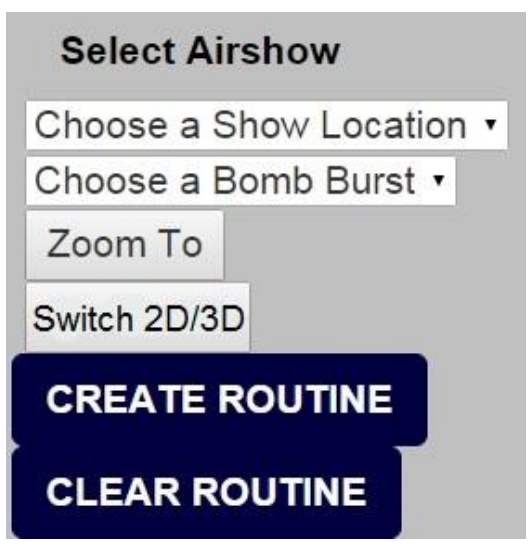




\section{D Visualization}

The entire airshow routine can be visualized in 3D. Clicking the Switch 2D/3D button will toggle between the two views. By default, the application will load in $2 \mathrm{D}$. Once the button is pressed, the $3 \mathrm{D}$ visualization will begin to load in the map viewer. In this view normal basic controls still apply, and the user can 'fly' through the environment. All layers are automatically added to this view. Mountains and buildings will be extruded to actual real-world height. Airshow locations points are set to 1,000ft in elevation. 